\title{
Self-Renewal and Differentiation of Reactive Astrocyte-Derived Neural Stem/Progenitor Cells Isolated from the Cortical Peri-Infarct Area after Stroke
}

\author{
Issei S. Shimada, Matthew D. LeComte, Jerrica C. Granger, Noah J. Quinlan, and Jeffrey L. Spees \\ Department of Medicine, Stem Cell Core, University of Vermont, Colchester, Vermont 05446
}

In response to stroke, subpopulations of cortical reactive astrocytes proliferate and express proteins commonly associated with neural stem/progenitor cells such as glial fibrillary acidic protein (GFAP) and Nestin. To examine the stem cell-related properties of cortical reactive astrocytes after injury, we generated $G F A P-C r e E R^{T M}$; $t d R F P$ mice to permanently label reactive astrocytes. We isolated cells from the cortical peri-infarct area $3 \mathrm{~d}$ after stroke, and cultured them in neural stem cell medium containing epidermal growth factor and basic fibroblast growth factor. We observed tdRFP-positive neural spheres in culture, suggestive of tdRFP-positive reactive astrocyte-derived neural stem/progenitor cells (Rad-NSCs). Cultured Rad-NSCs self-renewed and differentiated into neurons, astrocytes, and oligodendrocytes. Pharmacological inhibition and conditional knock-out mouse studies showed that Presenilin 1 and Notch 1 controlled neural sphere formation by Rad-NSCs after stroke. To examine the self-renewal and differentiation potential of Rad-NSCs in vivo, Rad-NSCs were transplanted into embryonic, neonatal, and adult mouse brains. Transplanted Rad-NSCs were observed to persist in the subventricular zone and secondary Rad-NSCs were isolated from the host brain $28 \mathrm{~d}$ after transplantation. In contrast with neurogenic postnatal day 4 NSCs and adult NSCs from the subventricular zone, transplanted Rad-NSCs differentiated into astrocytes and oligodendrocytes, but not neurons, demonstrating that Rad-NSCs had restricted differentiation in vivo. Our results indicate that Rad-NSCs are unlikely to be suitable for neuronal replacement in the absence of genetic or epigenetic modification.

\section{Introduction}

Neural stem cells (NSCs) are multipotent cells that self-renew and differentiate into neurons, astrocytes, and oligodendrocytes (Reynolds and Weiss, 1992, 1996; Morshead and van der Kooy, 2004; Neumeister et al., 2009). NSCs reside as astrocyte-like cells in neurogenic niches such as the subventricular zone (SVZ) of the lateral ventricle, and the subgranular zone (SGZ) of the hippocampus (Ihrie and Alvarez-Buylla, 2008). Although NSCs in neurogenic niches have been well studied, whether stem/progenitor cells reside in non-neurogenic niches, such as the cortex, remains under debate (Gould et al., 1999; Palmer et al., 1999; Kornack and Rakic, 2001; Nunes et al., 2003).

Astrocytes are one of the major cell types in the CNS, and occupy $20-50 \%$ of brain volume in mammals. Astrocytes contribute to numerous homeostatic functions in the CNS (Nedergaard et al., 2003; Sofroniew, 2009). Glial fibrillary acidic

Received Aug. 22, 2011; revised March 15, 2012; accepted April 20, 2012.

Author contributions: I.S.S. and J.L.S. designed research; I.S.S., M.D.L., J.C.G., and N.J.Q. performed research; I.S.S. contributed unpublished reagents/analytic tools; I.S.S. and J.L.S. analyzed data; I.S.S. and J.L.S. wrote the paper.

This work was supported by National Institutes of Health (P20 RR016435). I.S.S. was supported by a postdoctoral fellowship from the American Heart Association (10P0ST3730026).We thank Dr. Suzanne Baker for GFAP-CreER ${ }^{T M}$ mice (St. Jude Children's Research Hospital, Memphis, TN) and Dr. Hans Joerg Fehling for ROSA-tdRFP mice (University Clinics UIm, Ulm, Germany). We thank M. Wadsworth, C. Charland, and H. Shimada for technical help.

The authors declare no competing financial interests.

Correspondence should be addressed to Dr. Jeffrey L. Spees, Department of Medicine, Stem Cell Core, University of Vermont, 208 South Park Drive, Suite 2, Colchester, VT, 05446. E-mail: Jeffrey.Spees@uvm.edu.

DOI:10.1523/JNEUROSCI.4303-11.2012

Copyright $\odot 2012$ the authors $\quad 0270-6474 / 12 / 327926-15 \$ 15.00 / 0$ protein (GFAP) is often used as a marker to identify astrocytes in the cortex, and also astrocyte-like NSCs in neurogenic niches. Although NSCs and astrocytes express this marker in common, GFAP-expressing adult cortical astrocytes of uninjured animals do not have stem/progenitor cell properties (Laywell et al., 2000).

Following diverse CNS injuries, astrocytes are activated and described as "reactive astrocytes" (Sofroniew and Vinters, 2009; Robel et al., 2011). The appearance of reactive astrocytes is one of the most obvious phenomena observed after brain or spinal cord injury. Subpopulations of reactive astrocytes differentially express several stem cell-associated proteins such as Sox2, GFAP, Nestin, and RC2 (Pekny and Pekna, 2004; Buffo et al., 2008; Shimada et al., 2010; Zawadzka et al., 2010). We and others have demonstrated that locally-derived NSC-like cells can be generated from cortical tissues following injuries such as stroke, brain stab injury, or laser injury (Buffo et al., 2008; Nakagomi et al., 2009; Sirko et al., 2009; Shimada et al., 2010; Nakagomi et al., 2011; Robel et al., 2011). Following brain stab injury, it was shown that GLAST-positive reactive astrocytes could dedifferentiate, and form multipotent spheres in culture (Buffo et al., 2008). Therefore, reactive astrocytes appear to have greater plasticity than was previously realized, and may provide a source of multipotent cells or a cellular target for regenerative medicine. Importantly, however, the signaling pathway(s) that govern the formation of injury-induced NSCs from cortical tissue remain unidentified. In addition, whether reactive astrocyte-derived NSCs are multipotent following transplantation back to the CNS is not known. 
Here we use lineage tracing to determine the cellular origin of cortical neural sphere-producing cells after stroke. We demonstrate the isolation of reactive astrocyte-derived-NSCs (RadNSCs) from the cortical peri-infarct area $3 \mathrm{~d}$ following distal middle cerebral artery occlusion (dMCAO). Through pharmacological inhibition and conditional knock-out mouse studies, we show that Presenilin 1 and Notch 1 regulate the generation of Rad-NSC spheres from cortical peri-infarct tissues. Following transplantation of Rad-NSCs back to CNS, Rad-NSCs differentiated into astrocytes and oligodendrocytes. However, we did not observe neuronal differentiation, suggesting that unmodified Rad-NSCs are restricted from neuronal differentiation in vivo, despite their ability to produce neurons in culture.

\section{Materials and Methods}

Mice. All animal work was approved by the University of Vermont College of Medicine's Office of Animal Care in accordance with the American Association for Accreditation of Laboratory Animal Care and National Institutes of Health guidelines. Adult male C57BL/6J mice (6-8 weeks of age) were obtained from Taconic Farms. We obtained GFAP-CreER ${ }^{T M}$ mice from Dr. Suzanne Baker (St. Jude Children's Research Hospital, Memphis, TN) (Chow et al., 2008). The GFAP-CreER ${ }^{T M}$ mice express CreER under control of the $2.2 \mathrm{~kb}$ human GFAP ( $h G F A P$ ) promoter. We obtained ROSA26$t d R F P$ mice from Dr. Hans Joerg Fehling (University Clinics Ulm, Germany) (Luche et al., 2007). C57BL/6- Tg(UBC-GFP)30Scha/J mice (GFP mice, Cat\#

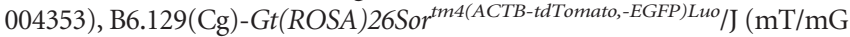
mice, Cat\# 007676), B6;129P-Psen $1^{\mathrm{tm} 1 \mathrm{Vln}} / \mathrm{J}$ mice (Presenilin 1-flox mice, Cat\# 007605), and Notch $1^{\mathrm{tm} 2 \mathrm{Rko}} / \mathrm{GridJ}$ mice (Notch 1-flox mice, Cat\# 006951) were obtained from The Jackson Laboratory. Genotyping was performed by PCR using REDExtract-N-Amp Tissue PCR Kit (XNAT-1KT; Sigma) following the manufacturer's instructions. We used the following primers: Notch 1, 5' -TGCCCTTTCCTTAAAAGTGG-3', 5' -GCCTACTCC GACACCCAATA- $3^{\prime}$; $t d R F P, 5^{\prime}$-AAGACCGCGAAGAGTTTGTCC- $3^{\prime}, 5^{\prime}$ TAAGCCTGCCCAGAAGACTCC-3', 5'-AAGGGAGCTGCAGTGGAG TA-3'; Presenilin 1, 5' -CAGGCACACTCACCCACAGA-3', 5' -GAAAATCA TATCCCCTACACTA- $3^{\prime}$; and GFAP-CreER ${ }^{T M}, 5^{\prime}$-AGCGATCGCTGCCA GGAT-3', 5'-ACCAGCGTTTTCGTTCTGCC-3'.

$d M C A O$ surgery. Adult mice (6-8 weeks) were anesthetized with isoflurane (1-5\%; Webster Veterinary) and body temperature was maintained with a heated pad. Focal cerebral ischemia was produced by permanently occluding the middle cerebral artery (MCA) (Bakondi and Shimada et al., 2009; Shimada et al., 2010). Under low-power magnification, the left temporo-parietal region of the head was shaved, and an incision was made between the left orbit and the left ear in the shape of a "U." An incision was made superiorly on the upper margin of the temporal muscle forward. The MCA was observed through the semitranslucent skull. A small burr hole (1-2 mm) was drilled into the outer surface of the skull just over the MCA. The inner layer of skull was removed with fine forceps, and the dura was opened with a cruciate incision. The MCA was then encircled with 10-0 monofilament nylon suture (7V33; S \& T), ligated, and transected superior to the ligation point. The small flap of facial skin was closed with Vetbond (3M Animal Care). For transgenic mouse studies, mice received $0.1 \mathrm{mg} / \mathrm{g}$ of tamoxifen (TM; T5648; Sigma) or corn oil by intraperitoneal injection for 3 consecutive days from day 0 immediately following dMCAO. Mice were euthanized 3 or $28 \mathrm{~d}$ after dMCAO. Brains were perfused with $4 \%$ paraformaldehyde (PFA), fixed in $4 \%$ PFA overnight at $4^{\circ} \mathrm{C}$, and then incubated in $30 \%$ sucrose for $2 \mathrm{~d}$ at $4^{\circ} \mathrm{C}$. Brains were mounted with OCT compound, and cut into $20-30 \mu \mathrm{m}$ frozen sections.

To label cells migrating from the lateral ventricle to the peri-infarct area after stroke, stereotaxic surgery was performed immediately after dMCAO surgery. Stereotaxic surgery was used to deliver $50 \mu \mathrm{mol} / \mathrm{l} \mathrm{DiI}$ (CellTracker CM-DiI; Invitrogen) in $5 \mu$ of PBS into the lateral ventricle (lateral ventricle coordinates: anteroposterior $[\mathrm{A} / \mathrm{P}]-0.3 \mathrm{~mm}$, mediolateral $[\mathrm{M} / \mathrm{L}] \pm 1.0 \mathrm{~mm}$, and dorsal/ventral [DV] $-3.0 \mathrm{~mm}$ ). The solution was delivered at a rate of $1 \mu \mathrm{l} / \mathrm{min}$. Three days later, mice were euthanized.

Isolation of Rad-NSCs and typical NSCs. For isolation of Rad-NSCs, brains were removed $3 \mathrm{~d}$ after $\mathrm{dMCAO}$, placed into a polyacrylic brain block (Acrylic Matrices, RBMA-200C; World Precision Instruments), and cut coronally in $1 \mathrm{~mm}$ sections. The sections were transferred into alpha-MEM (Invitrogen) in 6-well plates. The cortical peri-infarct area was dissected under a light microscope. Tissues from the anterior lateral ventricle, posterior lateral ventricle, hippocampal arch, dentate gyrus, white matter, and third ventricle were carefully excluded. The tissues containing the peri-infarct area were harvested, diced into $1 \mathrm{~mm}^{3}$ pieces, and digested in enzyme digestion solution containing $200 \mathrm{U}$ papain (Worthington), $20 \mu \mathrm{g} / \mathrm{ml}$ DNase (Worthington), $1.5 \mathrm{~mm}$ EDTA (Fisher Scientific), $1.5 \mathrm{~mm} \mathrm{CaCl}_{2}$ (Sigma), $2 \mathrm{mg}$ L-cysteine (Sigma), and DMEM/ F12 media (Invitrogen), at $37 \mathrm{C}^{\circ}$ for $15 \mathrm{~min}$. Following enzymatic digestion, the tissues were triturated two times in trituration solution: $10 \mathrm{ml}$ DMEM/F12 medium containing $15 \mathrm{mg}$ trypsin inhibitor (Invitrogen) and $15 \mathrm{mg}$ bovine serum albumin (BSA; Fisher Scientific). Cells were resuspended in DMEM/F12 medium containing 0.9 M sucrose and centrifuged to remove myelin $(111 \times g$ for $10 \mathrm{~min})$. Cells were grown at a low density $(10,000$ cells $/ \mathrm{ml})$ in NSC growth medium containing $10 \mathrm{ng} / \mathrm{ml}$ epidermal growth factor (EGF; BD Biosciences), $20 \mathrm{ng} / \mathrm{ml}$ basic fibroblast growth factor (bFGF; recombinant human bFGF produced in our laboratory), $1 \times$ B27 supplement (Invitrogen), $2 \mathrm{~mm}$ L-glutamine (Mediatech), and $100 \mathrm{U} / \mathrm{ml}$ penicillin/100 $\mu \mathrm{g} / \mathrm{ml}$ streptomycin, in Neurobasal A (Invitrogen) in $100 \mathrm{~mm}^{2}$ dishes (Nunc Thermo Fisher Scientific). Two weeks after plating, the number of neural spheres was counted under microscopy. To generate clonally-derived spheres, individual small spheres (10-100 cells) were pipetted under microscopy into separate 24 -well plates a week after plating. The clonally-isolated neurospheres were expanded and passaged in NSC growth medium for further studies. For sphere formation assays, clonally-isolated primary spheres were dissociated and plated again into NSC growth medium (24-well plates, 20 cells/ $\mu$ l density, $500 \mu \mathrm{l}$ per well). Seven days after plating, the number of neural spheres was counted under microscopy.

Postnatal day 4 neural stem cells (PD4-NSCs) and adult NSCs from the SVZ (adult-NSCs) were isolated as neurospheres from whole brains or the lateral ventricle of C57BL/6J mice, GFP mice, or $\mathrm{mT} / \mathrm{mG}$ mice (Neural Stem Cell Isolation Kit; StemCell Technologies). To isolate typical adult NSCs, $1 \mathrm{~mm}$ coronal brain sections from adult mice $(4-8$ weeks of age) were dissected to obtain tissues from the lateral ventricle and hippocampus under microscopy. To isolate NSCs from the lateral ventricle, tissues were obtained from the dorsal and lateral sides of the lateral ventricle. To isolate NSCs from the hippocampus, tissues were obtained from the hippocampus that excluded the posterior lateral ventricle.

Cell transplantation. Rad-NSCs were dissociated, diluted to 25,000 cells/ $\mu \mathrm{l}$ in Neurobasal A media, and $5 \mu \mathrm{l}$ of cell suspension was transplanted into the lateral ventricle $(\mathrm{A} / \mathrm{P},-0.3 \mathrm{~mm}, \mathrm{M} / \mathrm{L} \pm 1.0 \mathrm{~mm}$, and $\mathrm{D} / \mathrm{V}-3.0 \mathrm{~mm}$ ), hippocampus (A/P, $-2.3 \mathrm{~mm}, \mathrm{M} / \mathrm{L} \pm 1.3 \mathrm{~mm}$, and $\mathrm{D} / \mathrm{V}$ $-2.0 \mathrm{~mm}$ ), or rostral migratory stream (RMS; A/P, $-2.5 \mathrm{~mm}, \mathrm{M} / \mathrm{L} \pm 0.8$ $\mathrm{mm}$, and $\mathrm{D} / \mathrm{V}-3.0 \mathrm{~mm}$ ) in adult $\mathrm{C} 57 \mathrm{BL} / 6 \mathrm{~J}$ mice. Two microliters of the NSC cell suspension was transplanted into the brains of day $0-4$ C57BL/6J mice. For neonatal transplants, neonates were anesthetized with cold vapor from dry ice (carbon dioxide). The length between the posterior part of the left and right eyes was measured and divided into three equidistant sections. Rad-NSCs were injected at both edges of the middle section, one-third of the distance inward from the posterior part of left and the right eyes, and $\sim 1 \mathrm{~mm}$ below the skin using a 30 gauge needle (7803-07; Hamilton) and a $10 \mu \mathrm{l}$ Hamilton syringe (1701; Hamilton). To examine retention of transplanted cells and their location, some mice were injected with DiI (50 $\mu \mathrm{mol} / \mathrm{l}$, Invitrogen) or NSCs and the location of DiI or cell injection was analyzed. Neonatal mice were returned to their mothers after the cell injections. They were euthanized $28 \mathrm{~d}$ after injection. For embryonic transplants, $1 \mu \mathrm{l}$ of the Rad-NSC cell suspension was transplanted into the brains of embryonic day 15 (E15) C57BL/6J mice in utero. Rad-NSCs were injected at both edges of the middle section of the skull, one-third of the distance inward from the dorsal part of left and the right eyes, and $\sim 1 \mathrm{~mm}$ below the skin using a 30 gauge needle and a $10 \mu \mathrm{l}$ Hamilton syringe. The mother was returned to the cage, and pups were euthanized at E20 for further analysis.

5 -Bromo-2'-deoxyuridine administration. Mice received $50 \mathrm{mg} / \mathrm{kg}$ of BrdU (5-bromo-2' -deoxyuridine; Sigma) by intraperitoneal injection for 5 consecutive days from day 0 immediately after $\mathrm{dMCAO}$ or cell 
transplant. Mice were euthanized on day 28 after dMCAO or on day 28 or day 90 after cell transplant.

2, 3, 5-Triphenyltetrazolium chloride staining. Three days following $\mathrm{dMCAO}$, mice were euthanized. Brains were removed and coronal sections were cut to $1 \mathrm{~mm}$ thickness with a polyacrylic brain block. The sections were incubated in $10 \%$ 2, 3, 5-triphenyltetrazolium chloride (TTC; Sigma) solution (diluted in distilled water) for $15 \mathrm{~min}$ at $37^{\circ} \mathrm{C}$. After staining, the sections were photographed with a digital camera.

Treatment of cultured cells. Twenty-four well dishes (Nunc) were coated with $5 \mu \mathrm{g} / \mathrm{ml}$ laminin/poly-D-lysine (both from BD Biosciences) for $1 \mathrm{~h}$ or overnight. Cells $\left(5000\right.$ cells $/ \mathrm{cm}^{2}$ ) were plated into NSC growth medium in 24-well plates. One day after plating, the cells were incubated in various treatments. For the gamma-secretase inhibitor (GSI) study, cells were treated with L685-485 (Calbiochem) or dimethylsulfoxide (DMSO; vehicle) for $2 \mathrm{~d}$. For the 4-OH-TM study, cells were treated with $0.5 \mu \mathrm{M}$ 4-OH-TM (Sigma) or ethanol (vehicle) for $2 \mathrm{~d}$.

CyQUANT assay. Cells (5000 cells $/ \mathrm{cm}^{2}$ ) were grown in NSC medium in 24-well plates. Two days after plating, the cells were washed with PBS and plates were frozen at $-80^{\circ} \mathrm{C}(n=4$ for each time point). Cell numbers were quantified by dye labeling of nucleic acids (CyQUANT; Invitrogen) using a fluorescence plate reader (Synergy HT; BioTek Instruments).

MTS assay. Cells (5000 cells $/ \mathrm{cm}^{2}$ ) were grown in NSC medium in 24 -well plates. The cell number was assayed $2 \mathrm{~d}$ after plating (CellTiter 96 AQueous One Solution Cell Proliferation Assay; Promega). Relative intensity was quantified using a fluorescence plate reader.

Neural stem/progenitor cell differentiation. Cover glasses $12 \mathrm{~mm}^{2}$ round (Electron Microscopy Sciences) were coated with $5 \mu \mathrm{g} / \mathrm{ml}$ laminin/poly-Dlysine in 24-well plates for $1 \mathrm{~h}$. Clonally-isolated single dissociated cells (5000 cells $/ \mathrm{cm}^{2} / 500 \mu$ l passage $3-5$ ) were plated into 24 -well plates, and cultured in NSC growth medium for $1 \mathrm{~d}$. For differentiation, single dissociated cells were cultured in differentiation medium ( $1 \%$ fetal bovine serum, $100 \mathrm{U} / \mathrm{ml}$ penicillin $/ 100 \mu \mathrm{g} / \mathrm{ml}$ streptomycin, B27 supplement, $2 \mathrm{~mm}$ L-glutamine in Neurobasal A) for $7 \mathrm{~d}$. The number of cells that differentiated into neurons, astrocytes, and oligodendrocytes was determined after immunocytochemistry. To examine the multipotency of clonal spheres, the clonal spheres were plated into 24-well plates and cultured in NSC growth medium for $1 \mathrm{~d}$. For differentiation, the spheres were cultured in differentiation medium for $7 \mathrm{~d}$.

Reverse transcriptase PCR assays. Total RNA was prepared from Rad-NSCs and PD4-NSCs with a commercially available kit, according to the manufacturer's protocol (PureLink RNA Mini Kit; Invitrogen). DNase I (Turbo DNase; Invitrogen) was used to remove genomic DNA from RNA samples. cDNA was synthesized from $1 \mu \mathrm{g}$ of total RNA with SuperScript III RT (Invitrogen) and an oligo-dT primer. As a negative control, no reverse transcriptase (RT) samples were made by omitting SuperScript III from duplicate RT reactions. The CDNA was used for PCR with the following primers: Sox2, 5'-GGCAGCTACAGCATGATGCAGGAGC-3' and 5'-CTGGTC ATGGAGTTGTACTGCAGG-3';Pax6, 5' -AGTTCTTCGCAACCTGGC TA- ${ }^{\prime}$ and $5^{\prime}$-TGAAGCTGCTGCTGCTGATAGGA-3'; Mash $1,5^{\prime}$-CG TCCTCTCCGGAACTGA- $3^{\prime}$ and $5^{\prime}$-TCCTGCTTCCAAAGTCCA TT-3'; Olig2, 5' -TCATCTTCCTCCAGCACCTC-3' and 5' -CCGTAGAT CTCGCTCACCAG-3'; and GAPDH, $5^{\prime}$-CGGAGTCAACGGATTTGG TCGTAT- ${ }^{\prime}$ and 5'-AGCCTTCTCCATGGTGGTGAAGAC-3'.

Real-time quantitative RT-PCR. Real-time quantitative PCR (qRT-PCR) was performed using SYBR Green I detection Taq ReadyMix (S4438; Sigma) containing $5 \mathrm{~mm} \mathrm{MgCl}_{2}$ and $400 \mathrm{~nm}$ of each primer in a final $24 \mu \mathrm{l}$ reaction volume. We ran reactions with an Applied Biosystems 7500 Fast Real TimePCR system (Applied Biosystems). The cDNA was used for PCR with the following primers: GAPDH, 5'-CGGAGTCAACGGATTTGGTCGTAT-3' and $5^{\prime}$-AGCCTTCTCCATGGTGGTGAAGAC-3'; Hes1, 5'-TGAAGGAT TCCAAAAATAAAATTCTCTGGG- $3^{\prime}$ and $5^{\prime}$ - CGCCTCTTCTCCATGA TAGGCTTTGATGAC-3'; Deltex1, 5' -TGAGGATGTGGTTCGGAGGT-3' and 5' -CCCTCATAGCCAGATGCTGTG-3'; and RBPJ, 5' $^{\prime}$-TGGCACTGTT CAATCGCCTT- ${ }^{\prime}$ ' and $5^{\prime}$-AATCTTGGGAGTGCCATGCCA- ${ }^{\prime}$.

Flow cytometry analysis. Cells $(500,000$ cells $/ \mathrm{ml})$ were suspended in 0.5 $\mathrm{ml}$ alpha-MEM and analyzed by closed stream flow cytometry (Epics XL, Beckman Coulter; LSR II, Becton Dickinson).

Magnetic-activated cell sorting. To isolate GLAST-positive cells from whole brains, we performed magnetic-activated cell sorting (MACS) us- ing GLAST antibody (130-095-822; Miltenyi Biotec) according to the manufacturer's instructions.

Immunoblotting. Total proteins were extracted from the sorted cells with a cell lysis buffer (PBS with $0.1 \%$ SDS, $1 \times$ Complete protease inhibitor cocktail tablets; Roche). Protein concentrations were determined with a Bio-Rad DC Protein Assay (Bio-Rad). Protein preparations were separated by SDS-PAGE and transferred to an immobilon-P membrane (PVDF; Millipore). The membrane was blocked with 5\% BSA (BP1605; Fisher Scientific) and then incubated with a primary antibody. After washing, the membrane was incubated with a horseradish peroxidaseconjugated secondary antibody for $1 \mathrm{~h}$ at room temperature. The blot was visualized with Western Lightning Plus-ECL (NEL103001EA; Pierce), and exposed to Kodak x-ray film. Equivalence of protein loading was verified by probing for GAPDH or $\beta$-actin. The antibodies used were against GFAP (Z0334; DAKO), NICD1 (Notch 1 intracellular domain, ab8925; Abcam), Presenilin 1 (MA1-752; Thermo Scientific), GAPDH (MAB374; Millipore), and $\beta$-Actin (A5441; Sigma).

Immunohistochemistry. Frozen sections were dried for $30 \mathrm{~min}$ at $37^{\circ} \mathrm{C}$, and incubated in PBS for $15 \mathrm{~min}$. Sections were then blocked in blocking buffer (10\% normal goat serum, $0.4 \%$ Triton $\mathrm{X}$ in PBS) for $1 \mathrm{~h}$ at room temperature. For $\mathrm{BrdU}$ staining, sections were treated with $2 \mathrm{~N} \mathrm{HCl}$ for $30 \mathrm{~min}$ at $37^{\circ} \mathrm{C}$ and washed before the incubation with blocking buffer. For galactocerebroside (GalC) staining, sections were treated with proteinase $\mathrm{K}\left(20 \mu \mathrm{g} / \mathrm{ml}\right.$; Invitrogen) for $5 \mathrm{~min}$ at $37^{\circ} \mathrm{C}$. Sections were incubated in the following primary antibodies overnight at $4^{\circ} \mathrm{C}$ : $\beta$-tubulin III (1:1000, PRB-435P, Tuj1; Covance), BrdU (1:500, A21300; Invitrogen), BrDU (1:500, ab6326, Abcam), Calbindin (1:1000, CB38; Swant), Calretinin (1:1000, AB149; Millipore), doublecortin (DCX; 1:1000, AB5910; Millipore), GalC (1:200, MAB342; Millipore), GFAP (1:1000, Z0334; DAKO), GFAP (1:500, G3893; Sigma), green fluorescent protein (GFP; 1:1000, A11122; Invitrogen), GST $\pi$ (1:1000, MSA-102E; Stressgen), Hu C/D (1:1000, A21271; Invitrogen), NG2 (1:200, MAB5384; Millipore), NG2 (1:200, AB5320; Millipore), Nestin (1:500, PRB-315C; Covance), Nestin (1:500, ab81755; Abcam), NeuN (1:1000, MAB377; Millipore), NICD1 (1:500, ab8925; Abcam), RC2 (1:40, University of Iowa Hybridoma Bank), red fluorescent protein (RFP; 1:250, 632496; Clontech), Sox2 (1:1000, AB5603; Millipore), S100B (1:500; S2532; Sigma), and tyrosine hydroxylase (TH; 1:1000, TYH; Aves Labs). Antibodies against RFP (1: 250, 5F8; Chromotek) and Ki67 (1:500, ab1667; Abcam) were incubated overnight at room temperature. After three PBS washes, sections were incubated in secondary antisera (Alexa Fluor 488 or Alexa Fluor 594, 1:500; Invitrogen) for $1 \mathrm{~h}$ at room temperature. Cell nuclei were stained with DAPI (Vector Laboratories). Isotype controls: rabbit IgG $(2 \mu \mathrm{g} / \mathrm{ml})$, mouse $\operatorname{IgG}(2 \mu \mathrm{g} / \mathrm{ml})$, and mouse $\operatorname{IgM}(2 \mu \mathrm{g} / \mathrm{ml})$ were used as negative controls for immunohistochemical stains as in Shimada et al. (2010). Photomicrographs were obtained using an epifluorescence deconvolution microscope with an automated $x, y, z$ stage (Leica DM6000B; Leica) and Leica FW4000 software. For deconvolution, images were obtained every $0.1 \mu \mathrm{m}$ under $100 \times$ magnification, and deconvolved using Leica Deblur software. For some photomicrographs, images were obtained using a confocal microscope (Zeiss LSM 510 META; Zeiss Microimaging).

Immunocytochemistry. Cells were fixed with 4\% PFA for $15 \mathrm{~min}$ at room temperature and washed three times with PBS. Some cells were treated with methanol to eliminate endogenous GFP or tdRFP expression. Cells were blocked as above, and incubated with primary antisera overnight at $4^{\circ} \mathrm{C}$ : Nestin (1:1000; Covance), RC2 (1:40; University of Iowa Hybridoma Bank), Sox2 (1:1000; Millipore Bioscience Research Reagents), $\beta$-tubulin III (1:1000, PRB-435P, TUJ1; Covance), and GFAP (1:500; Sigma). Nonfixed, live cells were stained for O4 (1:50, O7139; Sigma) and then postfixed for analysis. After three PBS washes, cells were incubated in secondary antisera for $1 \mathrm{~h}$ at room temperature. The cover glasses were mounted onto slides with DAPI or Citifluor (100496-532; VWR International).

Cell quantification and statistics. The infarct area was defined by tissue autofluorescence, whereas the peri-infarct area was defined by the presence of GFAP-positive reactive astrocytes. One of every ten sections was quantified. Cell counts were performed by observers blinded to slide (sample) identity. Cell numbers were determined with the Optical Fractionator of Stereo Investigator (MBF Bioscience) in the cortical periinfarct area. Stereo Investigator was used in conjunction with a Nikon 

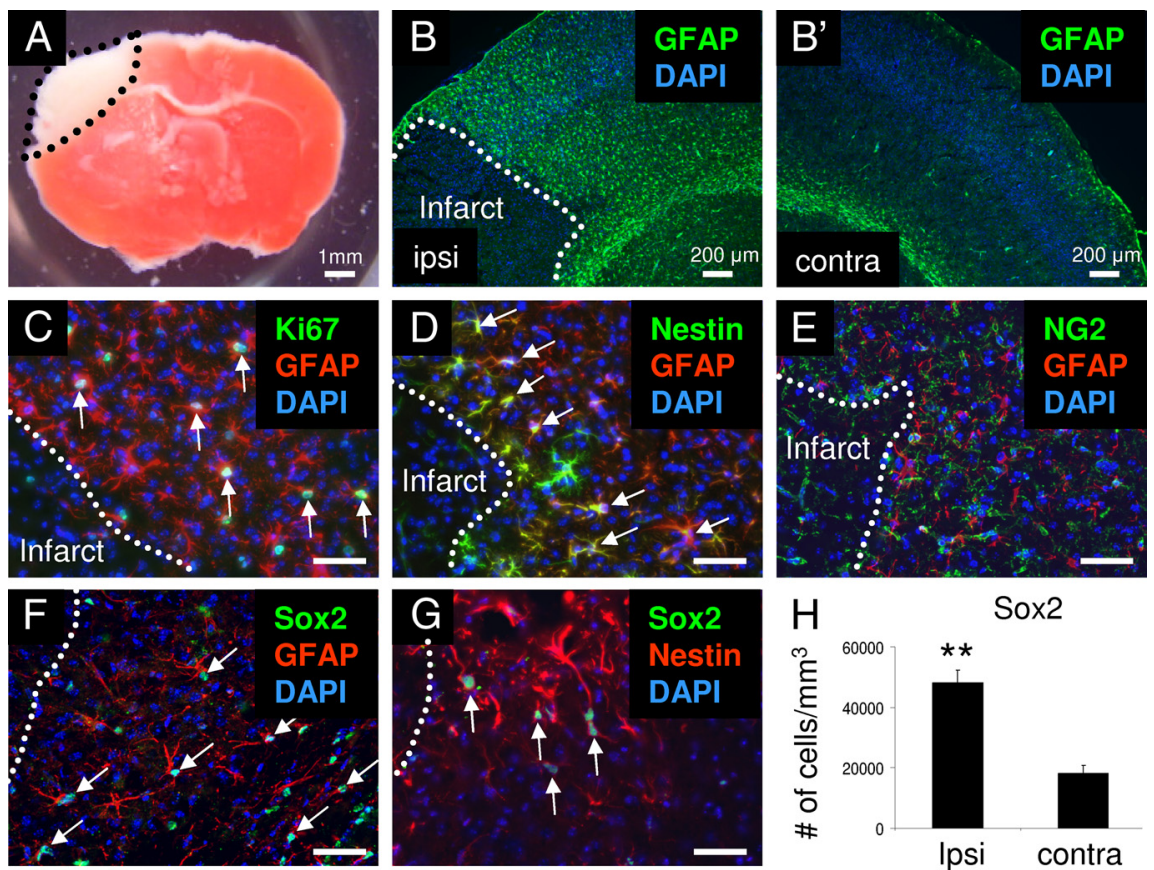

Figure 1. Emergence of reactive astrocytes in the peri-infarct area after stroke. $A$, Representative TTC staining of an infarct in $[57 \mathrm{BL} / 6$ mouse brain. The infarct region is white. $\boldsymbol{B}, \boldsymbol{B}^{\prime}, \mathrm{GFAP}$-positive reactive astrocytes (green) were observed surrounding the infarct area on the ipsilateral side of the cortex, but not on the contralateral side of the cortex. $C-E$,Some GFAP-positive reactive astrocytes (red) expressed Ki67 ( $\boldsymbol{C}$, green) and Nestin ( $\boldsymbol{D}$, green), but did not express NG2 ( $\boldsymbol{E}$, green) in the peri-infarct area. $\boldsymbol{F}, \boldsymbol{G}$, GFAP- and Nestin-positive reactive astrocytes (red) expressed Sox2 (green). $\boldsymbol{H}$, The number of Sox2-positive cells was significantly greater on the ipsilateral side of the cortex compared with the contralateral side of the cortex after stroke. Arrows indicate colocalizations. Scale bars: $50 \mu \mathrm{m}$. Dotted lines, border of stroke infarct core. Cell nuclei are shown by DAPI staining (blue). ${ }^{* *} p<0.01$.

Optiphot 2 microscope, a Hitachi HVC20 camera, a Heidenhahn focus encoder, and a motorized computer-driven $X-Z$ stage (all microscope attachments provided by MBF Biosciences). Under low magnification, the area of interest was identified and boundary contours were drawn using the software-pointing device. Spacing between sampling sites (grid size) was set such that $6-12$ sampling sites were counted per section. For GFAP, RC2, Nestin, or S100B-positive cell quantification, cell identity was ascertained by DAPI localization. We defined the inner peri-infarct area as cortical tissue that covered $200 \mu \mathrm{m}$ radially outside the edge of the infarct core where proliferative reactive astrocytes were typically located (Shimada et al., 2010). All data were expressed as means and SEM. Comparisons made between any two groups were performed by two-tailed Student's $t$ test. Values of $p<0.05$ were considered significant.

\section{Results}

GFAP-positive reactive astrocytes express NSC-related proteins after cortical stroke

To study reactive astrocytes following stroke, we induced cortical infarcts by dMCAO (Shimada et al., 2011). Three days following stroke, mice were euthanized and brains were prepared for analysis. TTC staining revealed that the infarct caused by dMCAO occurred exclusively in the cortex, but not in the striatum (Fig. $1 A$ ). Immunohistochemical studies identified numerous GFAPpositive reactive astrocytes on the ipsilateral side of the cortical infarct area, but not on the contralateral side of the cortex (Fig. $\left.1 B, B^{\prime}\right)$. GFAP-positive reactive astrocytes adjacent to the infarct core expressed the cell proliferation marker, Ki67, and several NSC-associated proteins such as Nestin, RC2, and Sox2 (Fig. 1C-E) (Shimada et al., 2010). We did not observe Nestin or RC2 expression on the contralateral side of the brain after stroke. In agreement with recent studies, we observed that GFAP-positive reactive astrocytes did not express the oligodendrocyte progenitor cell marker, NG2 (Dimou et al., 2008; Barnabé-Heider et al.,
2010; Zawadzka et al., 2010). As reported, the NSC-associated protein, Sox2, was expressed by many reactive astrocytes and astrocytes in the cortex (Komitova and Eriksson, 2004). After stroke, we observed that GFAP-positive reactive astrocytes and Nestin-positive reactive astrocytes expressed Sox 2 in the peri-infarct area (Fig. $1 F, G)$. To examine whether stroke induced a greater number of cells that expressed NSC-associated proteins, we examined the number of Sox2-positive cells after stroke. We observed a significantly increased number of Sox2-positive cells on the ipsilateral side of the brain compared with the contralateral side of the brain $(n=3, p<0.01$; Fig. $1 H)$.

\section{Heterogeneous populations of reactive astrocytes are observed in the peri- infarct area of GFAP-CreER ${ }^{T M}$; $t$ dRFP mice after stroke}

To examine GFAP-positive reactive astrocytes, we generated GFAP-CreER ${ }^{T M}$; tdRFP mice. In GFAP-CreER ${ }^{T M} ; t d R F P$ mice, tdRFP was induced permanently in GFAP-positive cells after TM administration (Luche et al., 2007; Chow et al., 2008). Focal cortical stroke was induced by the dMCAO surgery on GFAPCreER ${ }^{T M}$; $t$ dRFP mice. Mice received 0.1 $\mathrm{mg} / \mathrm{g}$ of TM by intraperitoneal injection for 3 consecutive days starting immediately after stroke. Three days after $\mathrm{dMCAO}$, mice were euthanized and brains were prepared for analysis. tdRFP-positive cells were observed in the periinfarct area (Fig. 2A). By immunohistochemistry, $58.7 \%$ of GFAP-positive cells were positive for tdRFP, and $74.5 \%$ of tdRFP-positive cells expressed GFAP in the peri-infarct area of the cortex ( $n=3$ mice; Fig. $2 A$ ). To further analyze the characteristics of tdRFP-positive cells in the peri-infarct area, we stained for the presence of other proteins expressed by reactive astrocytes. Nestin is a marker of NSCs and neural progenitor cells, and its expression is observed in a subpopulation of reactive astrocytes after stroke (Shimada et al., 2010; White et al., 2010). Three days after stroke, $63.3 \%$ of Nestin-positive cells were positive for tdRFP, and $78.9 \%$ of tdRFP-positive cells expressed Nestin in the peri-infarct area of the cortex $(n=3$ mice; Fig. $2 B)$. RC2 is a commonly used marker of radial glial cells during development and is expressed by a subpopulation of reactive astrocytes after stroke that reside directly adjacent to the infarct core (Shimada et al., 2010). We observed that $41.5 \%$ of RC2-positive cells were positive for tdRFP, and $24.2 \%$ of tdRFP-positive cells expressed RC2 in the peri-infarct area of the cortex ( $n=3$ mice; Fig. $2 C$ ). S100B is a commonly used marker of astrocytes and reactive astrocytes (Sellers et al., 2009). We observed that $31.1 \%$ of S100B-positive cells were positive for tdRFP, and $28.4 \%$ of tdRFP-positive cells expressed S100B in the peri-infarct area of the cortex $(n=3$; Fig. $2 D)$.

\section{Isolation of tdRFP-positive spheres from the cortical peri-infarct area after stroke}

Previously, we reported the isolation of locally-derived NSC-like cells from the peri-infarct area $3 \mathrm{~d}$ after stroke (Shimada et al., 2010). In that work, we observed neural sphere formation from 

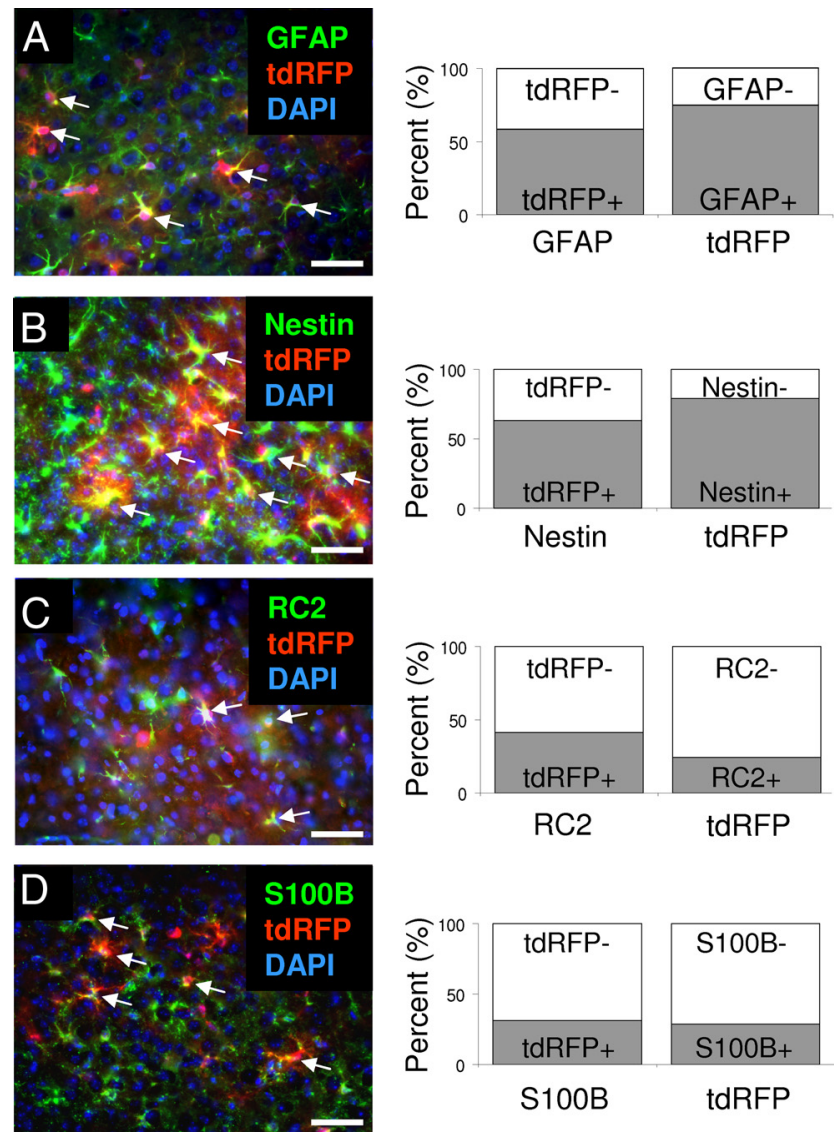

Figure 2. Heterogeneous tdRFP-positive reactive astrocytes in GFAP-CreER ${ }^{T M}$; $t d R F P$ mice. $\boldsymbol{A}-\boldsymbol{D}$, Three days after stroke and TM treatment, tdRFP-positive reactive astrocytes (red) expressed GFAP ( $\boldsymbol{A}$, green), Nestin ( $\boldsymbol{B}$, green), RC2 (C, green), and S100B ( $\boldsymbol{D}$, green) in the periinfarct area in GFAP-CreER ${ }^{T M}$; $t d R F P$ mice. The percentage of colocalization was quantified in the peri-infarct area. Arrows indicate colocalizations. Scale bars: $50 \mu \mathrm{m}$. Cell nuclei are shown by DAPI staining (blue).

peri-infarct tissues after stroke, but the cellular origin of the neural sphere-forming cells was not determined. To study the origin of locally-derived neural spheres after stroke, we isolated cells from the peri-infarct area of GFAP-CreER ${ }^{T M}$; $t$ dRFP mice $3 \mathrm{~d}$ following the $\mathrm{dMCAO}$ surgery (Fig. $3 A$ ). To prevent contamination of typical NSCs residing in neurogenic niches, tissues from the anterior lateral ventricle, posterior lateral ventricle, hippocampal arch, dentate gyrus, white matter, and third ventricle were carefully excluded from the dissected peri-infarct tissues. We cultured cells in NSC growth medium with EGF and bFGF. After 2 weeks in culture, we observed tdRFP-negative spheres and tdRFP-positive spheres ( $n=3$; Fig. $3 B, C)$. As a negative control, cortical tissues from the contralateral side of the brain were harvested. From the contralateral side of cortical tissues, we did not observe sphere formation $(n=3$; Fig. $3 D)$.

\section{Lack of neuroblast migration from the SVZ to the cortex after stroke}

To determine whether the cells that generated tdRFP-positive neural spheres had migrated from the SVZ after stroke, migrating neuroblasts were analyzed by immunohistochemistry. DCXpositive neuroblasts did not migrate into the peri-infarct area from the SVZ $3 \mathrm{~d}$ following stroke (Fig. $3 E, F$ ). Moreover, the staining pattern for DCX in the SVZ on the ipsilateral and contralateral sides of the brain was similar. Since there could be
DCX-negative neuroblasts migrating from the SVZ to the periinfarct area, we also stained to localize PSA-NCAM-positive immature neuroblasts. However, we did not observe PSA-NCAMpositive cells in the peri-infarct area $3 \mathrm{~d}$ following stroke (data not shown). To examine whether any cells from the lateral ventricle migrated into the peri-infarct area, DiI was injected into the lateral ventricle to label cells in the SVZ immediately after the dMCAO surgery. As a positive control for DiI labeling, we examined whether DiI-positive cells migrated in the RMS from the SVZ. We observed the migration of DiI-positive cells from the lateral ventricle to the RMS, demonstrating that our DiI injection was successful (data not shown). However, we found that DiI-positive cells did not migrate into the peri-infarct area $3 \mathrm{~d}$ after stroke (Fig. $3 G, H)$. These data indicated that cells from the lateral ventricle niche did not migrate into the cortical peri-infarct area within $3 \mathrm{~d}$ after MCA ligation in the $\mathrm{AMCAO}$ stroke model.

We also examined tdRFP-positive cells in the SVZ. Similar to a report that used the same GFAP-promoter strain (Chow et al., 2008), we found that most GFAP-positive cells on the striatal side of the lateral ventricle did not express tdRFP in GFAP-CreER ${ }^{T M}$; tdRFP mice (Fig. $3 I, J$ ). We then isolated cells from the dorsal and lateral (striatal) sides of the lateral ventricle $3 \mathrm{~d}$ after dMCAO, and cultured them in NSC growth medium. After 1-2 weeks of culture, numerous lateral ventricle-derived neural spheres were observed (Fig. $3 K, L)$. However, most spheres did not express tdRFP $(n=3)$. Therefore, the lack of tdRFP-labeled neural spheres from the lateral ventricle appeared to relate to mosaicism in the transgenic mouse model. As a positive control, cells from the hippocampus were also cultured. Many tdRFP-positive cells were observed in the SGZ of the hippocampus (data not shown). We then isolated cells from the hippocampus and cultured them in NSC growth medium. As expected, hippocampus-derived neural spheres expressed tdRFP (data not shown). In agreement with previous reports, we failed to passage the hippocampusderived neural spheres in culture (Seaberg and van der Kooy, 2002; Clarke and van der Kooy, 2011). Altogether, these data indicated that the tdRFP-positive neural spheres isolated from the cortical peri-infarct area after stroke had originated from tdRFP-positive reactive astrocytes of the peri-infarct area, and not from cells that had migrated from the SVZ. Hereafter, we call the injury-induced, tdRFP-positive neural sphere-forming cells reactive astrocyte-derived NSCs or Rad-NSCs.

\section{Rad-NSCs self-renew and are multipotent in culture}

To examine the self-renewal and multipotential capacities of Rad-NSCs in culture, clonal Rad-NSC spheres were derived. To obtain clonal spheres, individual spheres from low-density cultures were pipetted under microscopy into separate 24-well dishes. To confirm that the isolated clonal sphere population was homogenous in terms of tdRFP expression, we analyzed the passaged clonal spheres by flow cytometry. As a negative control, we used PD4-NSCs that were negative for tdRFP. The clonal tdRFPpositive sphere population was homogenous for tdRFP expression ( $n=2$; Fig. $4 A$ ). To examine the self-renewal capacity of Rad-NSCs ex vivo, sphere formation assays were performed with the clones. As a positive control, we used PD4-NSCs. As expected, PD4-NSCs formed secondary and tertiary spheres in culture. Clonal Rad-NSC spheres were also able to form secondary and tertiary spheres in culture. The number of Rad-NSC spheres was significantly less than that of cultures from PD4-NSCs, and the Rad-NSC spheres were smaller in size (number of spheres: secondary, $p<0.01$; tertiary, $p<0.01$; size of spheres: secondary, $p<0.01$; tertiary, $p<0.01, n=3$; Fig. $4 B, C)$. We observed that 

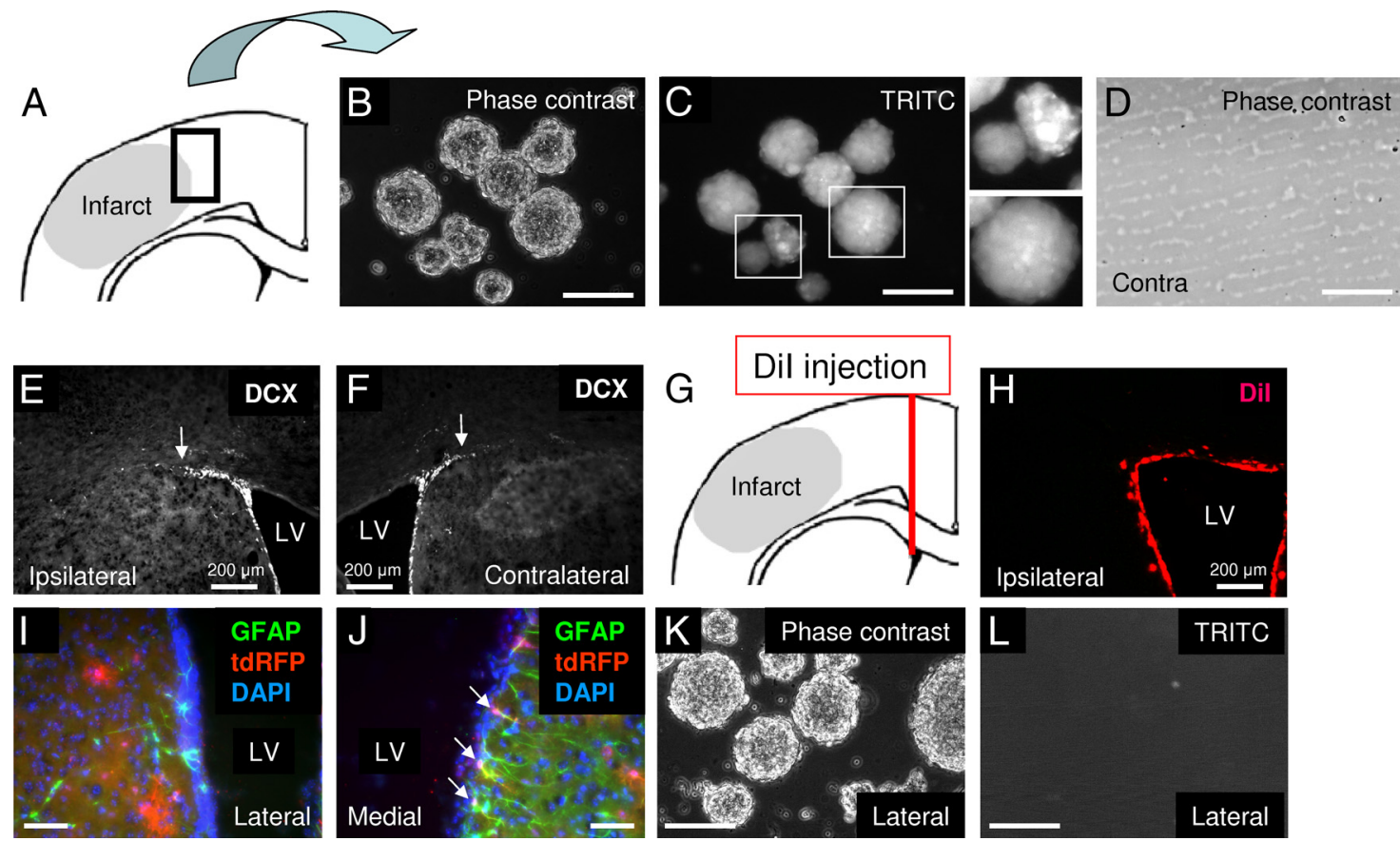

Figure 3. tdRFP-positive Rad-NSCs isolated from the cortical peri-infarct area. $A$, Schematic illustration of cell isolation from the cortical peri-infarct area $3 \mathrm{~d}$ after stroke. Tissue was dissected from the area delineated by the black box. $\boldsymbol{B}, \boldsymbol{C}$, Clonally-isolated cells formed neural spheres that expressed tdRFP (TRITC filter) in culture. $\boldsymbol{D}$, We were unable to generate neural spheres from tissues of the contralateral side of the cortex. $\boldsymbol{E}, \boldsymbol{F}$, Neuroblasts (DCX) did not migrate from the SVZ to the peri-infarct area in the cortex $3 \mathrm{~d}$ after stroke. Note that the relative staining areas for ipsilateral $(\boldsymbol{E})$ and contralateral $(\boldsymbol{F})$ DCX-positive cells did not differ. $\mathbf{G}$, Schematic illustration of Dil injection immediately after stroke to label migrating cells derived from the lateral ventricle. $\boldsymbol{H}$, Dil-labeled cells (red) did not reach the cortical peri-infarct area $3 \mathrm{~d}$ after stroke. I, J, Most GFAP-positive cells (green) on the lateral side of the lateral ventricle (LV) did not express tdRFP (red), but colocalization was observed in the medial side of the LV in GFAP-CreER ${ }^{T M}$; $t d R F P$ mice. $K, L$, Most neural spheres isolated from the dorsal and lateral sides of the LV of GFAP-CreER ${ }^{T M}$; $t d R F P$ mice did not express tdRFP. Cell nuclei are shown by DAPI staining (blue). Scale bars: $200 \mu \mathrm{m}$ where indicated; otherwise $50 \mu \mathrm{m}$.

Rad-NSCs grew slower than PD4-NSCs (day 2, $p<0.01$; day 4, $p<0.01$; day $6, p<0.01 ; n=4$; Fig. $4 D$ ). Rad-NSCs expressed several mRNAs commonly expressed by NSCs such as Sox2, Pax6, Mash1, and Olig2 (Fig. 4E). To determine whether Rad-NSCs also expressed NSC-associated proteins, we performed immunocytochemistry. Immunocytochemical analysis demonstrated that tdRFP-positive Rad-NSCs expressed several stem cell-associated proteins, such as Sox2, Nestin, RC2, and GFAP in culture (Fig. $4 F-J)$.

To examine the multipotency of Rad-NSCs, dissociated RadNSCs were differentiated for $7 \mathrm{~d}$. Immunocytochemistry demonstrated that cultured Rad-NSCs differentiated into neurons, astrocytes, and oligodendrocytes (Fig. $4 K-N$ ). Approximately $89 \%$ of Rad-NSCs differentiated into astrocytes. Since dissociated cell analysis does not fully examine the multipotency of spheres, we also used a more stringent test to examine multipotency. To examine the multipotency of Rad-NSC spheres and PD4-NSC spheres, clonal spheres were plated into 24-well dishes and differentiated for $7 \mathrm{~d}$ and then examined by triple immunocytochemistry (Fig. 4O-Q). Following immunocytochemistry, the number of unipotent (astrocytes), bipotent (astrocytes and neurons, or astrocytes and oligodendrocytes), and tripotent (astrocytes, neurons, and oligodendrocytes) spheres was quantified. Interestingly, Rad-NSC cultures contained significantly more tripotent spheres than did PD4-NSC cultures (unipotent, $p=0.32$; bipotent, astrocytes, and neurons, $p=0.10$; astrocytes and oligodendrocytes, $p=0.17$; tripotent, $p<0.05$; Fig. $4 Q$ ).

Control of Rad-NSC self-renewal by Presenilin 1- and Notch 1-dependent signaling

The Notch signaling pathway has critical roles in development and NSC maintenance. Notch is cleaved by gamma-secretase, and the intracellular domain is transported to the nucleus and interacts with DNA via DNA-binding proteins. Notch signaling maintains the undifferentiated state of NSCs, and also enhances gliogenesis from NSCs (Furukawa et al., 2000; Satow et al., 2001; Ge et al., 2002; Hitoshi et al., 2002; Iso et al., 2003; Eiraku et al., 2005; Alexson et al., 2006; Androutsellis-Theotokis et al., 2006; Patten et al., 2006; Borghese et al., 2010). Therefore, we examined whether reactive astrocytes in cortical peri-infarct tissues expressed the cleavage product of the Notch 1 receptor after stroke. By immunohistochemistry, GFAP-positive reactive astrocytes expressed NICD1 in the cortical peri-infarct area after stroke (Fig. 5) (Shimada et al., 2011). tdRFP-positive Rad-NSCs also expressed NICD1 in culture (Fig. 5A). Based on the requirement for Notch signaling to maintain NSCs, we hypothesized that this gamma-secretase-mediated pathway also regulates Rad-NSCs. First, to determine whether gamma-secretase-mediated cleavage was required for proliferation, cultured Rad-NSCs were plated and treated with a GSI for $2 \mathrm{~d}$. The number of RadNSCs decreased significantly following GSI treatment compared with vehicle control $(p<0.01$; Fig. $5 B)$, suggesting that a gamma secretase-mediated pathway regulated the proliferation of Rad-NSCs.

Presenilin 1 is thought to act as the catalytic subunit for gamma-secretase (Fortini, 2002). To determine whether gammasecretase itself was important for self-renewal of Rad-NSCs, we generated GFAP-CreER ${ }^{T M}$;Presenilin 1-inducible conditional knock-out (GP cKO) mice. In GP cKO mice, Presenilin 1 is deleted after TM treatment in GFAP-positive reactive astrocytes. To determine whether Presenilin 1 expression levels were decreased in astrocytes in GP cKO mice following TM treatment, we isolated astrocytes by MACS using an antibody against GLAST, a cell surface protein commonly expressed by astrocytes (Mori et al., 

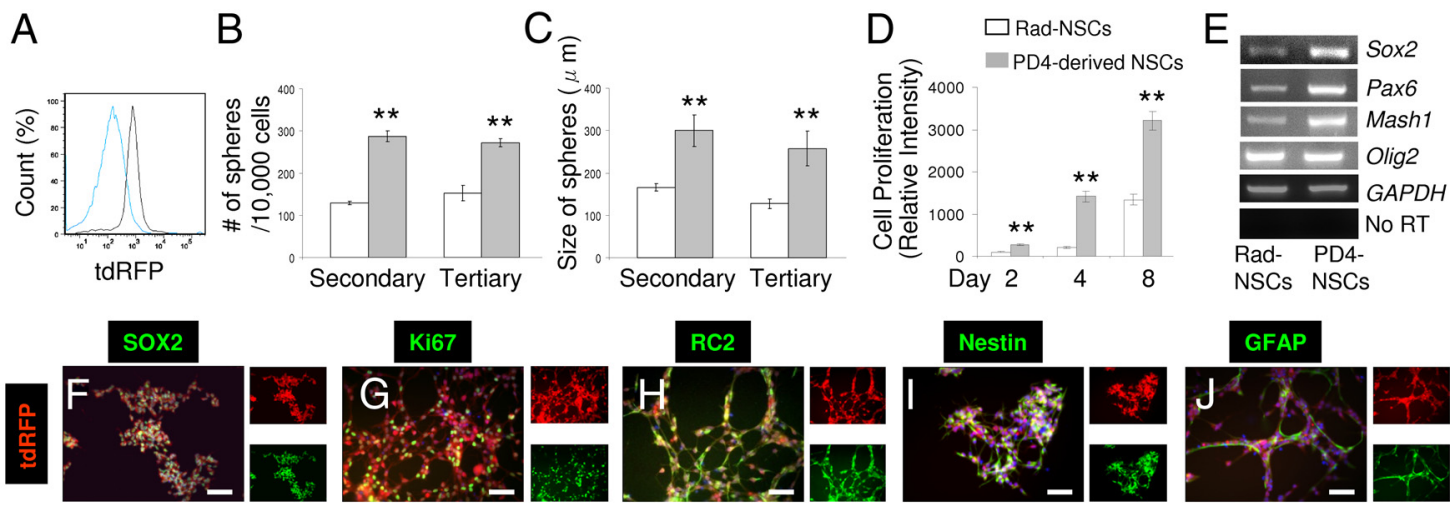

$$
\text { Nestin }
$$

GFAP
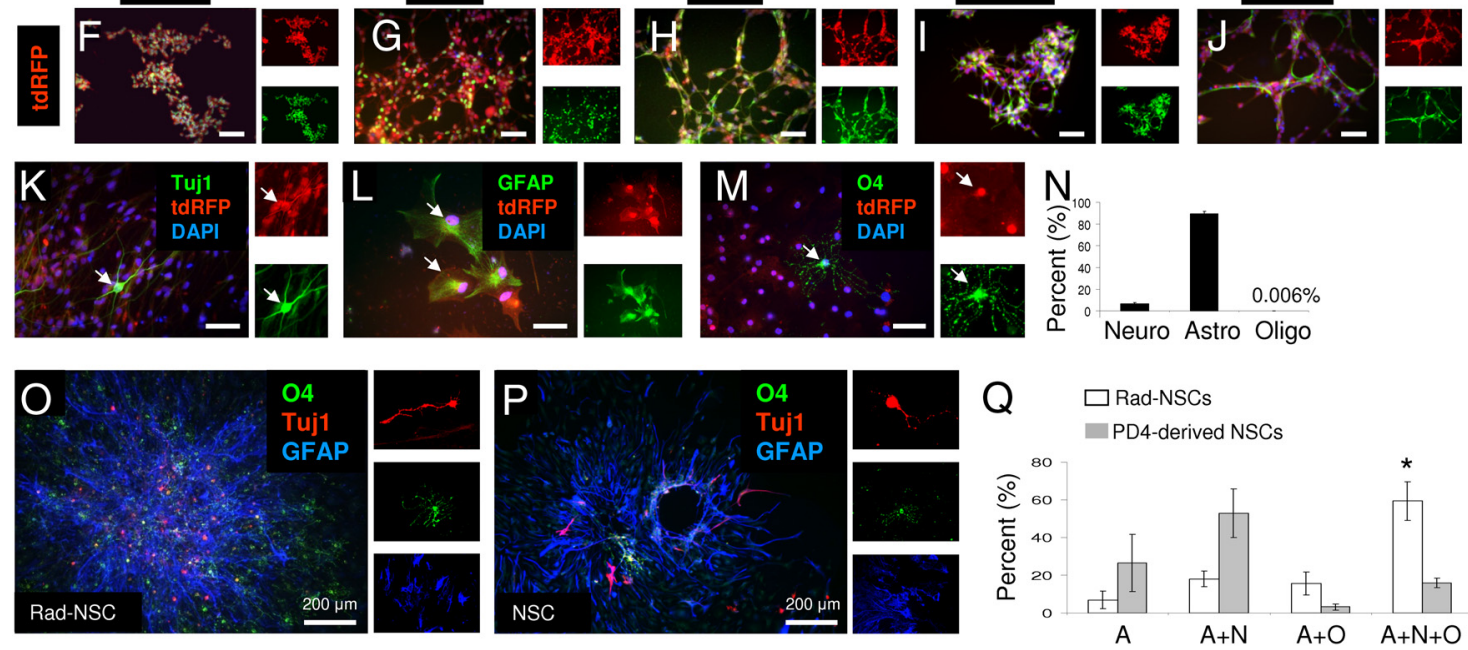

Figure 4. Cultured Rad-NSCs have characteristics of NSCs, self-renew, and are multipotent ex vivo. A, Clonally-isolated Rad-NSCs were homogenous in terms of tdRFP expression (gray). PD4-derived NSCS (blue) were used as a negative control. B, C, By sphere formation assays, Rad-NSCs formed fewer and smaller spheres compared with PD4-derived NSCs. D, Rad-NSCS proliferated at a slower rate compared with PD4-NSCS. E, By RT-PCR assays, Rad-NSCs expressed different transcription factor mRNAs commonly expressed by NSCS. F-J, Stem cell-related proteins and the cell proliferation marker, Ki67 (green), were also expressed by Rad-NSCs (tdRFP, red). $\boldsymbol{K}-\boldsymbol{N}$, Cultured Rad-NSCs (red) were dissociated and differentiated into Tuj1-positive neurons ( $\boldsymbol{K}$, green), GFAP-positive astrocytes ( $\boldsymbol{L}$, green), and 04-positive oligodendrocytes ( $\boldsymbol{M}$, green). Note that most Rad-NSCs differentiated into astrocytes. $\mathbf{0}-\mathbf{Q}$, Multipotency of clonal Rad-NSC spheres and clonal PD4-NSC spheres. O, P, Tuj1-positive neurons (red), 04-positive oligodendrocytes (green), and GFAP-positive astrocytes (blue) were stained by immunocytochemistry. Right, Provides magnified view of neurons (red), oligodendrocytes (green), and astrocytes (blue) in separate channels. $\mathbf{Q}$, The number of unipotent (A, astrocytes only), bipotent $(A+N$, astrocytes and neurons; $A+0$, astrocytes and oligodendrocytes), and tripotent ( $\mathrm{A}+\mathrm{N}+0$, astrocytes, neurons, and oligodendrocytes) spheres was quantified. Rad-NSCs produced more tripotent spheres than PD4-NSCs. $\boldsymbol{F}$ - $M$, Right, FITC and TRITC channels. Arrows indicate colocalizations. Cell nuclei are shown by DAPI staining (blue). Scale bars: $200 \mu \mathrm{m}$ where indicated; otherwise $50 \mu \mathrm{m}$. ${ }^{*} p<0.05$; ${ }^{* *} p<0.01$.

2006). We confirmed that sorted GLAST-positive cells expressed GFAP by Western blotting, indicating that GLAST-positive cells were enriched for astrocytes (Fig. 5C). GLAST-positive astrocytes were isolated and used for further analysis. We treated GP cKO mice with TM for $3 \mathrm{~d}$ and isolated astrocytes. By immunoblotting, we observed decreased Presenilin 1 expression levels in TMtreated mice compared with vehicle-treated mice $14 \mathrm{~d}$ after TM treatment but not $3 \mathrm{~d}$ after TM treatment, suggesting that residual Presenilin 1 was present in a proportion of GLAST-positive cells at $3 \mathrm{~d}$ after TM treatment (Fig. 5D; data not shown). It is noteworthy that by analysis of GFAP-CreER ${ }^{T M}$; $t$ dRFP mice, not all GFAP-positive cells expressed Cre enzyme (Fig. $2 A$ ). Therefore, we did not expect to observe knock-out of Presenilin 1 in all astrocytes of GP cKO mice. To determine whether reduction of Presenilin 1 affected neural sphere formation from reactive astrocytes after stroke, Rad-NSCs were isolated from cortical periinfarct tissues $3 \mathrm{~d}$ after stroke from GP cKO mice that were treated with TM for 3 consecutive days after MCA ligation. As negative controls, Rad-NSC spheres were isolated from the brains of GP cKO mice that were treated with corn oil, and also from littermate control mice (GFAP-CreER ${ }^{T M}$;Presenilin 1 heterozygous mice or Presenilin 1-loxp mice) that were treated with TM. After 2 weeks in culture, the number of neural spheres generated decreased significantly for GP cKO mice treated with TM compared with both negative control groups $(n=6-8$ for each group; Fig. 5E). To understand whether cell proliferation affected neural sphere formation from GP cKO-derived Rad-NSCs, we isolated clonal Rad-NSCs from GP cKO mice $3 \mathrm{~d}$ after stroke. Clonal Rad-NSCs were treated with either ethanol (vehicle control) or 4-OH-TM to flox Presenilin 1 in culture. Two days following treatment, the proliferation of Rad-NSCs was examined by CyQUANT assay and MTS assay. We observed that conditional knock-out of Presenilin 1 significantly reduced the proliferation of cultured Rad-NSCs ( $n=4$ for each group; Fig. $5 F, G$ ). Therefore, gamma-secretase-mediated signaling controlled both the generation of Rad-NSCs from cortical reactive astrocytes after stroke injury as well as the proliferation of Rad-NSCs in culture.

To determine whether Notch 1 signaling was important for self-renewal in Rad-NSCs, inducible GFAP-CreER ${ }^{T M}$;Notch 1 conditional knock-out ( $\mathrm{GN} \mathrm{cKO})$ mice were generated. In the GN cKO mice, Notch 1 is deleted after TM treatment in GFAPpositive reactive astrocytes. To examine Notch 1 expression levels in astrocytes, we performed Western blotting using sorted GLAST-positive cells. We observed decreased Notch 1 expression levels in TM-treated mice compared with oil-treated mice (Fig. $5 H$ ). Next, we examined expression of NICD1 in reactive astrocytes. By immunohistochemistry, we observed reduced NICD1 expression in reactive astrocytes in TM-treated mice, but not corn oil-treated mice $3 \mathrm{~d}$ after stroke (Fig. $5 I, J$ ). To determine whether Notch 1 reduction affected proliferation of reactive astrocytes, the number of proliferating reactive astrocytes was 

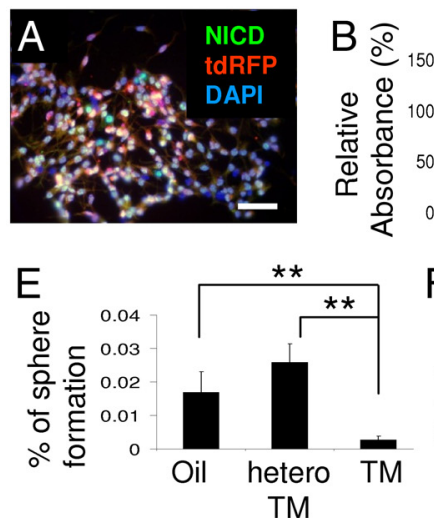

TM
C

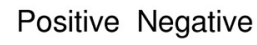

GFAP

$\beta$-Actin

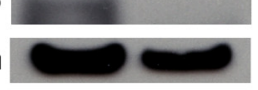

GSI
$\mathrm{F}$

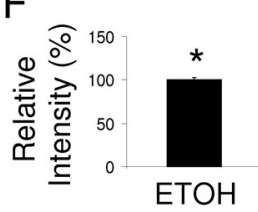

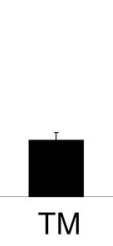

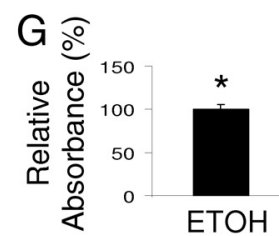

D

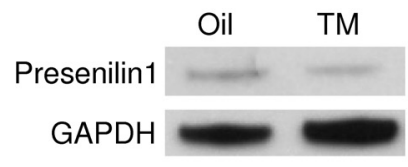

$\mathrm{H}$
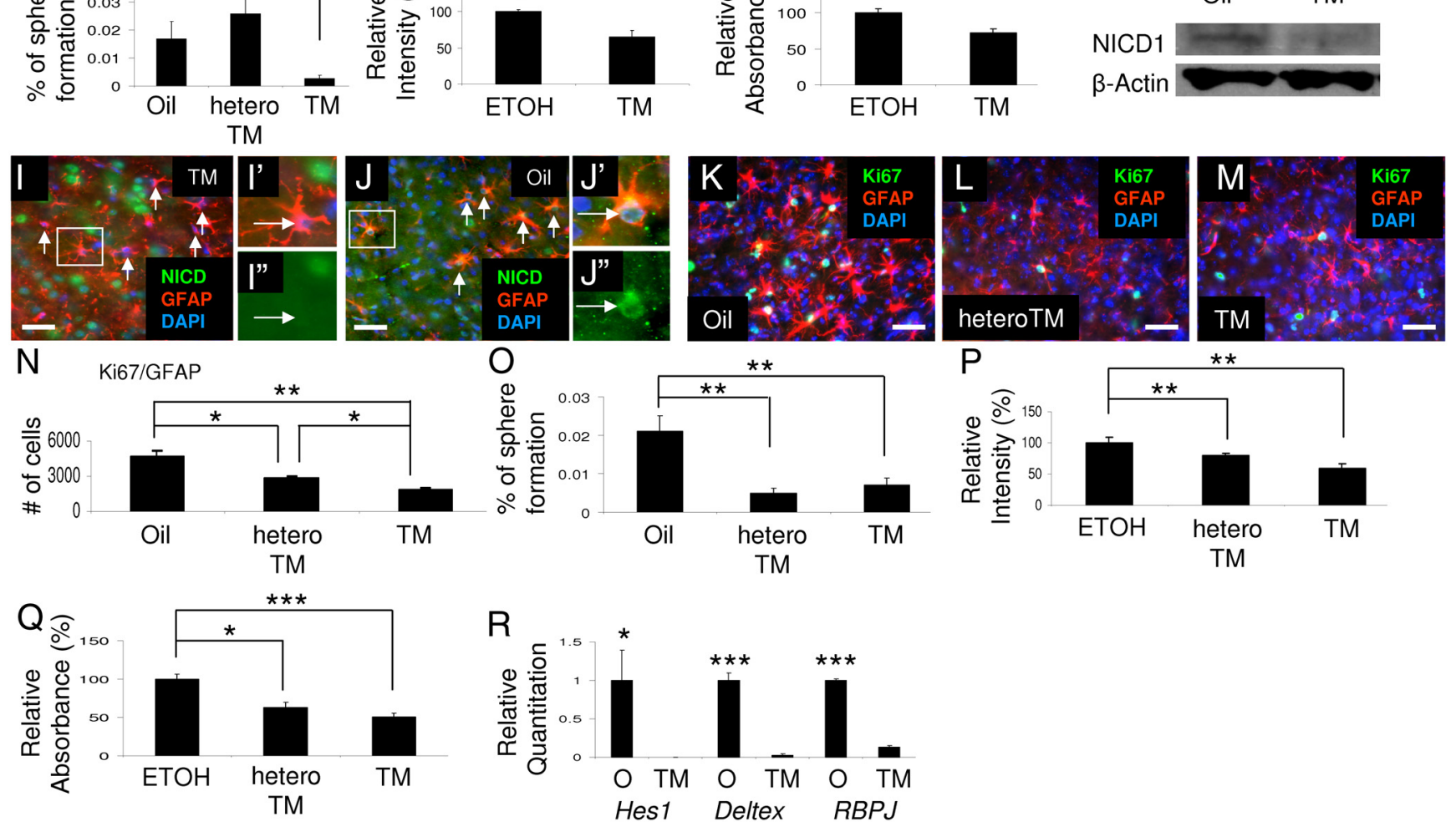

Figure 5. Notch 1 signaling regulates self-renewal of Rad-NSCS. A, NICD1 (green) was expressed in tdRFP-positive Rad-NSCS (red) in culture. $B$, GSI treatment reduced the number of Rad-NSCS compared with DMSO treatment. C, GLAST-positive cells were sorted from mouse brains by MACS. GLAST-positive cells were enriched with GFAP compared with GLAST-negative cells (as determined by immunoblotting). $\beta$-Actin levels were used as an internal loading control. $\boldsymbol{D}$, Following TM treatment of GFAP-CreER ${ }^{T M}$;Presenilin 1 cK0 mice (GP mice), Presenilin 1 expression levels decreased compared with levels in oil-treated GP mice. GAPDH was used as an internal loading control. $E$, TM treatment of GP mice significantly reduced the number of neural spheres formed in culture compared with control groups. $\boldsymbol{F}$ (CyQUANT assay), $\boldsymbol{G}$ (MTS assay): The number $(\boldsymbol{F})$ and growth $(\boldsymbol{G})$ of Rad-NSCs from GP mice were both significantly reduced following 4-0H-TM treatment compared with vehicle control. $\boldsymbol{H}$, Following TM treatment of GFAP-CreER ${ }^{T M}$; Notch 1 CKO mice (GN mice), NICD1 expression levels in sorted GLAST-positive cells were decreased compared with levels in control oil-treated mice. $\boldsymbol{I}, \boldsymbol{J}$, TM treatment of GN mice $(\boldsymbol{I})$, but not oil treatment $(\boldsymbol{J})$ removed NICD1 (green) expression in GFAP-positive reactive astrocytes (red). $\boldsymbol{K}-\boldsymbol{M}$, The numbers of Ki67 (green)-positive proliferating reactive astrocytes (GFAP, red) were compared in oil- or TM- treated GN mice and TM-treated GN heterozygous (hetero) mice. $\boldsymbol{N}$, TM treatment of GN mice and TM treatment of GN heterozygous mice significantly reduced the number of proliferating reactive astrocytes compared with oil treatment of GN mice. TM treatment of GN mice significantly reduced the number of proliferating reactive astrocytes compared with TM treatment of GN heterozygous mice. $\boldsymbol{O}$, TM treatment of GN mice and TM treatment of GN heterozygous mice significantly reduced the number of neural spheres formed in culture compared with oil treatment of GN mice. $P, \mathbf{Q}, 4-\mathrm{OH}-\mathrm{TM}$ treatment significantly reduced the proliferation of Rad-NSCs from GN mice and GN heterozygous mice compared with vehicle control (ETOH). $\boldsymbol{P}$, CyQUANT assay. $\mathbf{Q}$, MTS assay. $\boldsymbol{R}$, mRNA levels for proteins expressed downstream of Notch signaling were decreased in Rad-NSC spheres derived from TM-treated GN mice compared with oil-treated GN mice. Cell nuclei are shown by DAPI staining (blue). TM, tamoxifen; 0 , oil. Arrows indicate colocalizations. Scale bars: $50 \mu \mathrm{m} .{ }^{*} p<0.05 ;{ }^{* *} p<$ $0.01 ; * * * 0.001$.

quantified in the peri-infarct area $3 \mathrm{~d}$ following dMCAO. Three days after stroke, the number of total GFAP-positive astrocytes or the overall number of Ki67-positive proliferating cells did not differ among treatment groups (data not shown). However, in agreement with our previous study, the number of proliferating reactive astrocytes was significantly decreased in TM-treated GN cKO mice compared with TM-treated GN heterozygous cKO mice and the corn oil-treated group (Shimada et al., 2011). The number of proliferating reactive astrocytes was also significantly decreased in TM-treated GN heterozygous cKO mice compared with corn oil-treated mice of the same genotype ( $n=3-4$; Fig. $5 K-N$ ). These data indicate that Notch 1 knock-out affects reactive astrocyte proliferation in vivo.
To determine whether Notch 1 signaling affected neural sphere formation by reactive astrocytes after stroke, Rad-NSCs were isolated from cortical peri-infarct tissues of GN cKO mice that were treated with TM for 3 consecutive days after dMCAO. The number of neural spheres generated in culture decreased significantly for GN cKO mice treated with TM compared with corn oil-treated mice. Interestingly, the number of neural spheres was significantly decreased for GN heterozygous mice too $(n=4$; Fig. 5O). To understand whether cell proliferation affected neural sphere formation in GN cKO-derived Rad-NSCs, we isolated clonal Rad-NSCs from GN cKO mice $3 \mathrm{~d}$ after stroke. Clonal Rad-NSCs were treated with either ethanol or 4-OH-TM to reduce Notch 1 levels in cultured cells. Two days following treat- 

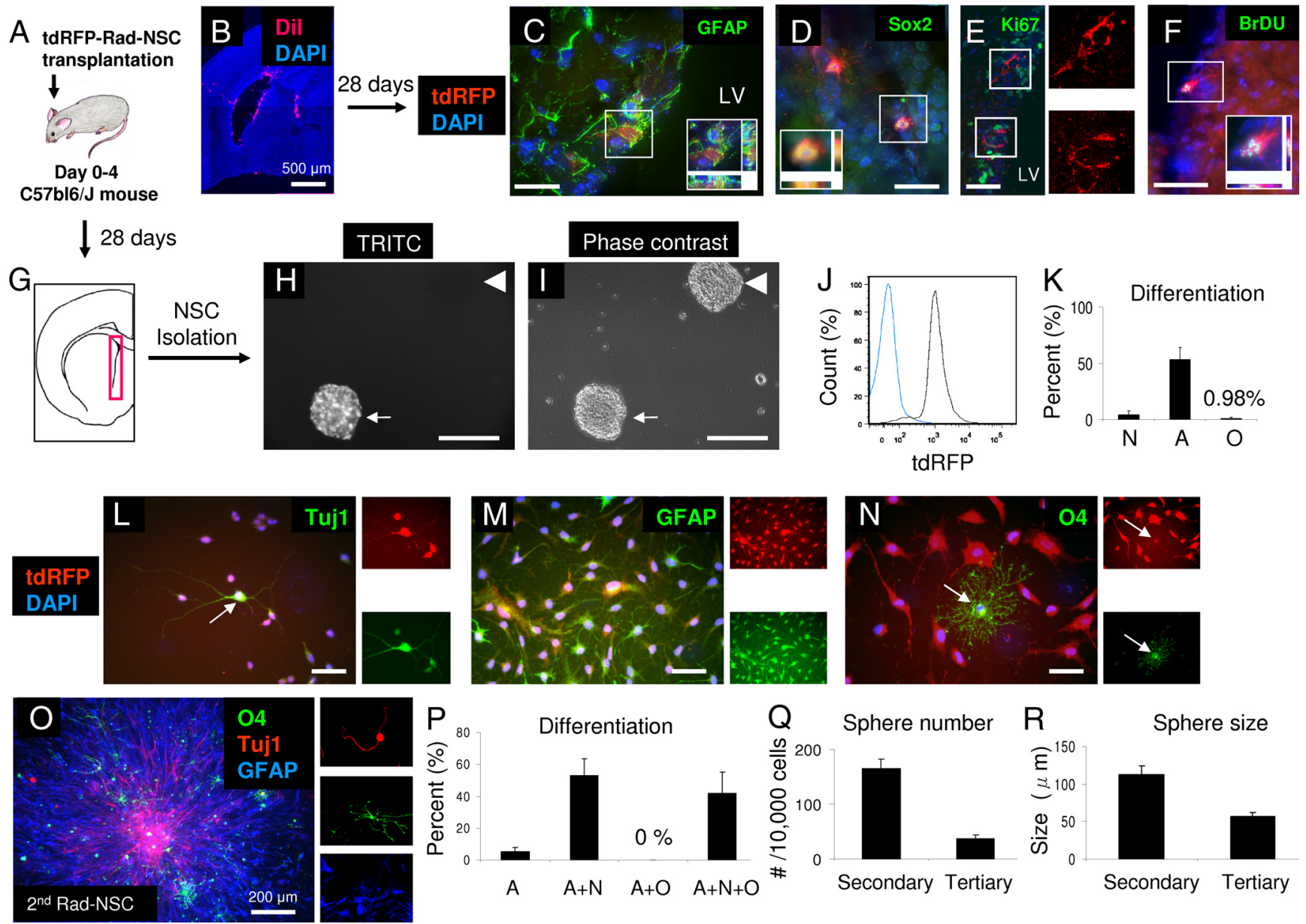

Figure 6. Self-renewal capacity of transplanted Rad-NSCs in vivo. A, Schematic illustration of tdRFP-positive Rad-NSC transplantation into PD0 - 4 C57BL/6 J mouse brain. B, Dil staining shown $4 \mathrm{~h}$ after transplantation of labeled cells into PDO mouse brain. Dil was observed in the lateral ventricle and the needle track. C $\boldsymbol{F}$, tdRFP-positive Rad-NSCs (red) located in the SVZ $28 \mathrm{~d}$ after transplantation. C, D, tdRFP-positive cells expressed stem cell markers, GFAP (C) and Sox2 (D).E, tdRFP-positive cells did not express Ki67, suggesting that these cells were not actively dividing. Right, Type B cell-like shape of engrafted tdRFP-positive cells. $\boldsymbol{F}$, tdRFP-positive cells expressed BrdU, suggesting that these cells self-renewed in the SVZ after transplantation. Insets (C, $\boldsymbol{D}, \boldsymbol{F})$, Deconvolution for colocalization ( $x, y, z$ planes). G, Schematic illustration of cell isolation from lateral ventricle $28 \mathrm{~d}$ after transplantation. $\boldsymbol{H}, \boldsymbol{I}$, Cells isolated from the SVZ generated both Rad-NSCderived, and typical C57BL/6J-derived neural spheres ex vivo. Arrow, Rad-NSC-derived neural sphere; arrowhead, host-derived neural sphere. J, Clonal secondary-isolated Rad-NSCS (second Rad-NSCs) were homogenous in terms of tdRFP expression (gray). PD4-derived NSCS (blue) were used as a negative control. $\boldsymbol{K}$, Most second Rad-NSCs differentiated into astrocytes. $\mathbf{L}-\boldsymbol{N}$, Second Rad-NSCs (red) differentiated into neurons $(\boldsymbol{L})$, astrocytes $(\boldsymbol{M})$, and oligodendrocytes $(\boldsymbol{N})$ ex vivo. Arrows indicate colocalizations. Right, FITC and TRITC channels. $\mathbf{0}, \boldsymbol{P}$, Tuj1-positive neurons (red), 04-positive oligodendrocytes (green), and GFAP-positive astrocytes (blue) were stained by immunocytochemistry. Right, Magnified views of neurons (red), oligodendrocytes (green), and astrocytes (blue) in separate channels. The numbers of unipotent ( $A$, astrocytes only), bipotent $(A+N$, astrocytes and neurons; $A+0$, astrocytes and oligodendrocytes), and tripotent $(A+N+0$, astrocytes, neurons, and oligodendrocytes) spheres were quantified. $\boldsymbol{Q}, \boldsymbol{R}$, Clonally-isolated second Rad-NSCs continued to form spheres ex vivo. Cell nuclei are shown by DAPI staining (blue). Scale bars: 500 or $200 \mu \mathrm{m}$ where indicated; otherwise $50 \mu \mathrm{m}$.

ment, the number of Rad-NSCs was examined. By CyQUANT assay and MTS assay, we observed that reduction of Notch 1 significantly reduced the number of cultured Rad-NSCs $(n=4$; Fig. 5P,Q). Therefore, Notch 1 signaling controlled both the generation of Rad-NSCs from reactive astrocytes as well as the proliferation of Rad-NSCs in culture. To examine whether downstream aspects of the Notch 1 signaling pathway was altered in TM-treated GN cKO-derived Rad-NSCs, Notch target gene mRNAs were examined by qRT-PCR. First, we confirmed that Notch 1 was deleted in Rad-NSCs derived from TM-treated GN mice by RT-PCR. We did not observe Notch 1 expression in Rad-NSCs derived from TM-treated GN mice (data not shown). qRT-PCR results also demonstrated that Hes 1, Deltex, and Rbpj mRNA levels were all significantly decreased in Rad-NSCs derived from TM-treated GN mice compared with corn oil-treated GN mice ( $n=3-6$; Fig. $5 R)$.

\section{Transplanted Rad-NSCs self-renew in vivo}

To study the self-renewal capacity of Rad-NSCs in vivo, tdRFPpositive Rad-NSCs were transplanted into day $0-4$ mouse brains (Fig. 6A). First, to examine retention of transplanted cells and their location, we injected DiI into the day 0 mouse brains. Four hours after injection, we observed DiI-positive cells located in the lateral ventricle. DiI-positive cells were also observed in the needle track (Fig. $6 B$ ). Twenty-eight days after transplantation, we observed tdRFP-positive cells residing in the lateral ventricle walls (Fig. $6 C-F$ ). We then investigated whether transplanted tdRFP-positive cells might reside in the lateral ventricle as NSCs. By Immunohistochemistry, tdRFP-positive cells in the SVZ expressed GFAP and Sox2 (Fig. 6C,D). However, we did not observe Rad-NSCs that expressed Ki67, suggesting that tdRFPpositive cells were not actively dividing at the time of the assay (Fig. 6E). To examine whether transplanted Rad-NSCs prolifer- 
ated in vivo, we injected BrdU to the mice for 5 consecutive days immediately after transplantation. We observed BrdU-positive Rad-NSCs in the SVZ 1 month after transplantation, indicating that tdRFP-positive Rad-NSCs proliferated in the SVZ after transplantation (Fig. 6F).

To examine whether Rad-NSCs maintained stem cell characteristics in the mouse brain after transplantation, we repeated transplantation of Rad-NSCs to day 0-4 mouse brains and dissected tissues from the lateral ventricle after $28 \mathrm{~d}$ (Fig. 6G). From these isolates, we observed neural sphere formation after 1 week of culture in NSC growth medium (Fig. $6 \mathrm{H}, I$ ). These data indicated that Rad-NSCs transplanted to the SVZ self-renewed and maintained their multipotency for differentiation. Notably, we observed both tdRFP-positive and tdRFP-negative neural spheres in culture, indicating that some neural spheres were obtained from host-derived NSCs of the SVZ, whereas some had derived from transplanted tdRFP-positive Rad-NSCs.

To determine the self-renewal capacity and multipotency of secondary-transplanted tdRFP-positive Rad-NSCs (hereafter called second Rad-NSCs), clonal tdRFP-positive spheres were isolated from mixed sphere cultures and analyzed. The purity of tdRFP cells in the clones was examined by flow cytometry. We found that clonally-isolated second Rad-NSCs were homogeneous in terms of tdRFP expression $(n=6$; Fig. $6 J)$. To confirm that second Rad-NSCs had self-renewal capacity and multipotency, sphere formation assays and differentiation assays were performed. Similar to primary Rad-NSCs, dissociated clonal second Rad-NSCs differentiated into neurons, astrocytes, and oligodendrocytes in culture (Fig. $6 \mathrm{~K}-\mathrm{N}$ ). To examine the multipotency of clonal second Rad-NSC spheres, spheres were plated into 24-well dishes and differentiated for $7 \mathrm{~d}$. Multipotency of clonal second Rad-NSC spheres was examined by immunocytochemistry (Fig. 6O). Following immunocytochemistry, the number of unipotent (astrocytes), bipotent (astrocytes and neurons, or astrocytes and oligodendrocytes), and tripotent (astrocytes, neurons, and oligodendrocytes) spheres was quantified (Fig. $6 P$ ). We observed that $42 \%$ of spheres from clonal second Rad-NSCs were tripotent. Clonal second RadNSCs also formed secondary and tertiary spheres, suggesting that second Rad-NSCs self-renewed ex vivo (Fig. 6Q,R). Therefore, the primary Rad-NSCs that were transplanted into neonatal brains maintained their ability to self-renew as NSCs in vivo for $28 \mathrm{~d}$.

\section{Transplanted Rad-NSCs generate astrocytes and oligodendrocytes in vivo}

To further examine the self-renewal and multipotency of tdRFPpositive Rad-NSCs in vivo, we first transplanted GFP-positive PD4-NSCs that were isolated from the lateral ventricles of healthy mice. For some transplants, the location of GFP-positive cells was examined $4 \mathrm{~h}$ after transplantation. As expected, we observed many GFP-positive PD4-NSCs in the lateral ventricle and the needle track (Fig. 7A). Other groups of transplanted mice were examined $28 \mathrm{~d}$ after transplantation. In these mice, many surviving GFP-positive cells were observed to remain in the SVZ after injection (Fig. 7B). Notably, however, we also observed GFPpositive cells in the olfactory bulb (Fig. $7 C$ ). To examine whether transplanted GFP-positive PD4-NSCs differentiated into neurons in vivo, confocal imaging was performed to visualize NeuN/ GFP-positive cells in the olfactory bulb. We found that $66.6 \%$ of GFP-positive cells differentiated into NeuN-positive neurons in the olfactory bulb ( $n=48$ cells, $n=4$ mice; Fig. $7 D$ ). We also observed 5\% GFP-positive cells, derived from PD4-NSCs, that differentiated into GFAP-positive astrocytes in the olfactory bulb ( $n=20$ cells, $n=2$ mice; Fig. $7 E$ ).
To examine whether adult NSCs were multipotent following transplantation, we isolated adult NSCs from the lateral ventricles of adult mice (6-8 weeks of age). We obtained adult NSCs from GFP mice and also from $\mathrm{mT} / \mathrm{mG}$ mice. Cells derived from $\mathrm{mT} / \mathrm{mG}$ mice express tdTomato without Cre recombination and provide a bright red signal to track cell lineage. We transplanted GFP-positive or tdTomato-positive cells into the lateral ventricles of neonatal mice (Fig. $7 F$ ). We observed migrating lineagetraced cells in the RMS 1 month after transplantation (Fig. 7G). Differentiated neurons and astrocytes derived from adult NSCs were also observed in the olfactory bulb. After 1 month, we found that $16.2 \%$ of transplanted adult NSCs differentiated into NeuNpositive neurons once they reached the olfactory bulb $(n=482$ cells, $n=3$ mice; Fig. $7 H$ ). We also observed that $14.1 \%$ of adult NSCs differentiated into GFAP-positive astrocytes in the olfactory bulb ( $n=377$ cells, $n=3$ mice; Fig. $7 I)$. These data confirmed that the multipotency of cultured adult NSCs could be evaluated by in vivo transplantation back to mouse brains (Neumeister et al., 2009).

We then examined the multipotency of transplanted tdRFPpositive Rad-NSCs. tdRFP-positive Rad-NSCs were transplanted into the RMS, lateral ventricle (SVZ), and hippocampi (SGZ) of adult C57BL/6J mice. Twenty-eight days after transplantation, brains were examined by immunohistochemistry. tdRFPpositive cells localized primarily to the injection areas in the RMS, SVZ, and SGZ ( $n=3-13$ mice; Fig. $8 A-C$ ). In adults, transplanted tdRFP-positive cells did not migrate to the RMS from the SVZ, and we did not observe tdRFP-positive cells in the olfactory bulb 1 month after transplantation. Moreover, after 1 month, no tdRFP-positive cells expressed NeuN (mature neuronal marker) in the striatum, SVZ, or SGZ, suggesting that the adult mouse brain did not provide a suitable environment for Rad-NSCs to differentiate into neurons.

To determine whether the age of the host affected the differentiation output from Rad-NSCs, tdRFP-positive Rad-NSCs were transplanted into PD0 -4 mouse brains ( $n=15$ mice). Following tdRFP-positive Rad-NSC transplantation, tdRFPpositive cells localized to the SVZ, gray matter, and white matter (Fig. 8D,E; data not shown). After $28 \mathrm{~d}$, transplanted tdRFP-positive Rad-NSCs differentiated into GFAP-positive astrocytes and GST $\pi$-positive oligodendrocytes in both gray and white matter $(n=85-198$ cells; Fig. $8 D-G)$. Although we observed tdRFP-positive cells in the SVZ, we did not observe any tdRFPpositive cells in the RMS or olfactory bulb. These data indicated that tdRFP-positive cells did not migrate into the olfactory bulb from the SVZ 1 month after transplantation into neonates. Additionally, tdRFP-positive cells were detected after direct transplantation to the hippocampus. However, analysis by confocal microscopy demonstrated that transplanted tdRFP-positive Rad-NSCs also did not differentiate into NeuN-positive mature neurons in the hippocampus (Fig. $8 F$ ). Similarly, we did not observe tdRFP-positive cells expressing NeuN in gray or white matter $28 \mathrm{~d}$ after transplantation into brains of neonates $(n=489$ cells; Fig. 8G). Since immature neuroblasts and some mature neurons do not express NeuN, we checked for other markers of neurogenesis. However, tdRFP-positive cells did not express other neuronal proteins, such as $\beta$-tubulin III, DCX, Hu C/D, or PSA-NCAM (data not shown). Therefore, tdRFPpositive Rad-NSCs differentiated into GFAP-positive astrocytes and GST $\pi$-positive oligodendrocytes, but not neurons 1 month after transplantation into neonates.

Because tdRFP-positive Rad-NSCs might require a longer time to differentiate into neurons, we also examined transplanted 


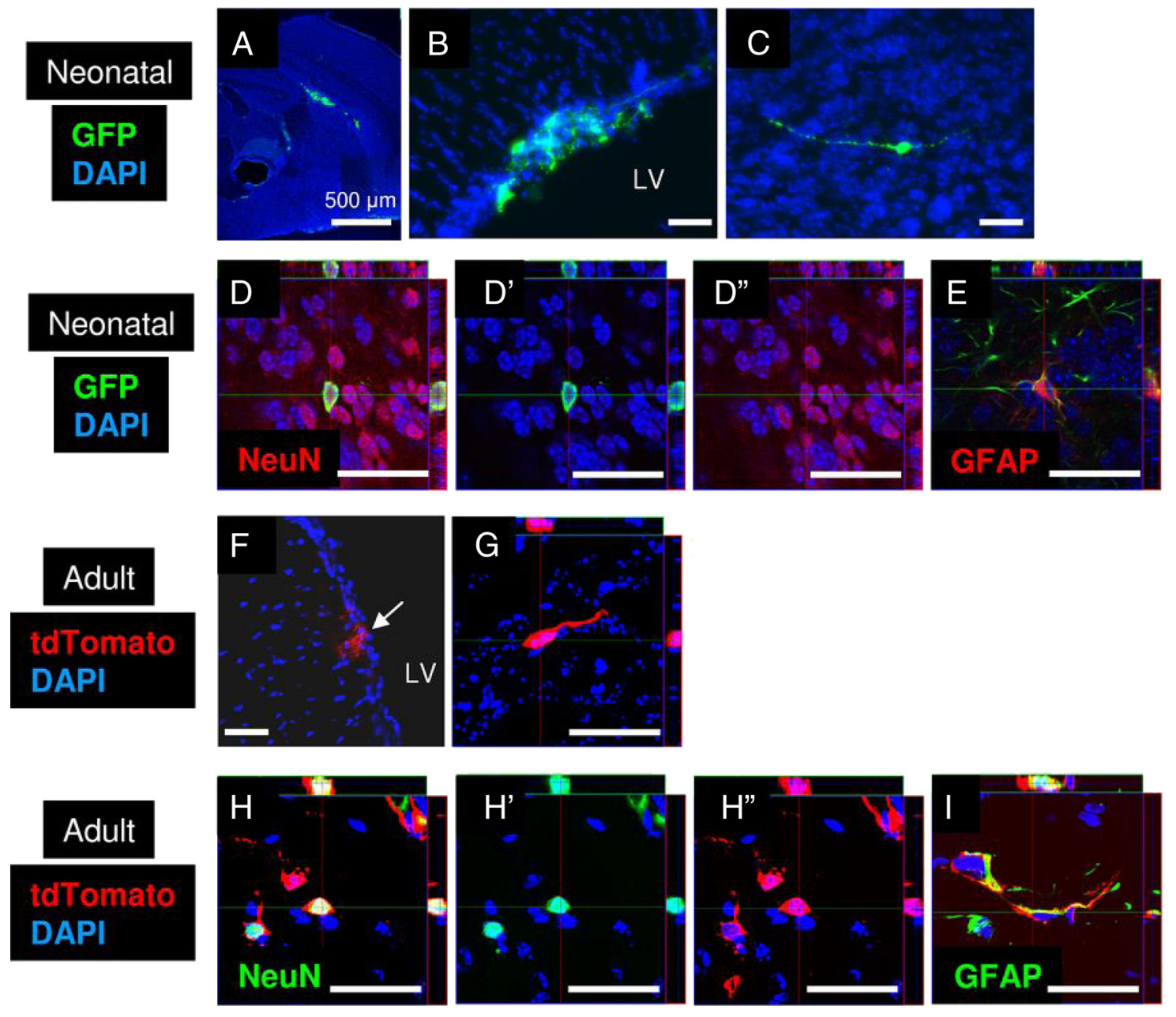

Figure 7. NSCs from the SVZ are multipotent and neurogenic in vivo after transplantation. A, GFP-positive PD4-NSCs (green) $4 \mathrm{~h}$ after transplantation into PDO mouse brains. B, GFP-positive cells (green) were observed in the lateral ventricle (LV) 1 month after transplantation. C, GFP-positive cells (green) with neuronal morphology were observed in the olfactory bulb 1 month after transplantation. $\boldsymbol{D}, \boldsymbol{E}$, Confocal imaging demonstrated that GFP-positive PD4-NSCs (green) differentiated into NeuN-positive neurons ( $\boldsymbol{D}$, red) and GFAP-positive astrocytes ( $\boldsymbol{E}$, red) in the olfactory bulb. $\boldsymbol{D}^{\prime}, \boldsymbol{D}^{\prime \prime}$, FITC and TRITC channels, respectively. $\boldsymbol{F}$, tdTomato-positive adult NSCS (red) derived from the SVZ were observed in the LV 1 month after transplantation. $\mathbf{G}$, tdTomato-positive cells (red) with the morphology of migrating neuroblasts were observed in the RMS 1 month after transplantation. $\boldsymbol{H}, \boldsymbol{I}$, tdTomato-positive adult NSCs (red) differentiated into NeuN-positive neurons ( $\boldsymbol{H}$, green) and GFAP-positive astrocytes ( $\boldsymbol{I}$, green) in the olfactory bulb 1 month after transplantation. $\boldsymbol{H}^{\prime}, \boldsymbol{H}^{\prime \prime}$, FITC and TRITC channels, respectively. Cell nuclei are shown by DAPI staining (blue). Scale bars: $500 \mu \mathrm{m}$ where indicated; otherwise $50 \mu \mathrm{m}$.

cells 3 months after transplantation into neonates. Three months after transplantation, tdRFP-positive cells expressed Nestin and Sox2 and also stained positive for BrdU in the SVZ, suggesting that self-renewing tdRFP-positive cells could reside in the SVZ for 3 months ( $n=4$ mice; Fig. $8 H-J$ ). Interestingly, at this time point, we observed that some tdRFP-positive cells had migrated to the olfactory bulb. However, immunohistochemistry showed that tdRFP-positive cells did not express NeuN in the olfactory bulb $(n=117$ cells; Fig. $8 \mathrm{~K})$. Since not all neurons in the olfactory bulb express NeuN, we also performed immunohistochemistry using other neuronal markers, such as $\beta$-tubulin III, DCX, TH, Calretinin and Calbindin (Parrish-Aungst et al., 2007; Batista-Brito et al., 2008). tdRFP-positive cells did not express any of these additional neuronal markers $(n=68-109$ cells; Fig. $8 \mathrm{~L}$; data not shown). In contrast, we observed that $21 \%$ of tdRFPpositive cells expressed GFAP. Thus, after 3 months, tdRFPpositive Rad-NSCs had migrated into the olfactory bulb and differentiated into astrocytes, but not neurons ( $n=123$ cells; Fig. $8 M)$. To better understand the identity of the Rad-NSC-derived cells in the olfactory bulb, we performed double stains for RFP and GalC (a marker of oligodendrocytes), NG2 (a marker of oligodendrocyte progenitor cells and pericytes), and smooth muscle $\alpha$-actin (SMA; a marker of smooth muscle cells and myofibroblasts). Despite confirmation of positive staining for each marker throughout the brain, we did not observe any tdRFPpositive cells that coexpressed GalC, NG2, or SMA (data not shown).

To determine whether the embryonic brain environment was more suitable for neuronal differentiation of Rad-NSCs, we transplanted tdRFP-positive Rad-NSCs in utero into E15 mouse brains. Mice were euthanized at E20 to examine neuronal differentiation. We observed many tdRFP-positive cells in both gray and white matter (Fig. $8 N$ ). Although tdRFPpositive cells were observed throughout the brains, transplanted tdRFP-positive Rad-NSCs did not differentiate into NeuN-, Hu C/D-, or $\beta$-tubulin III-positive neurons $(n=837$ cells, $n=5$ mice; Fig. $8 O, P$; data not shown). We observed that $53 \%$ of tdRFP-positive cells expressed GFAP in gray and white matter ( $n=548$ cells, $n=5$ mice; Fig. $8 Q$ ). Therefore, despite their ability to generate neurons in culture, we did not observe transplanted Rad-NSCs that differentiated into neurons in vivo, regardless of host age. 


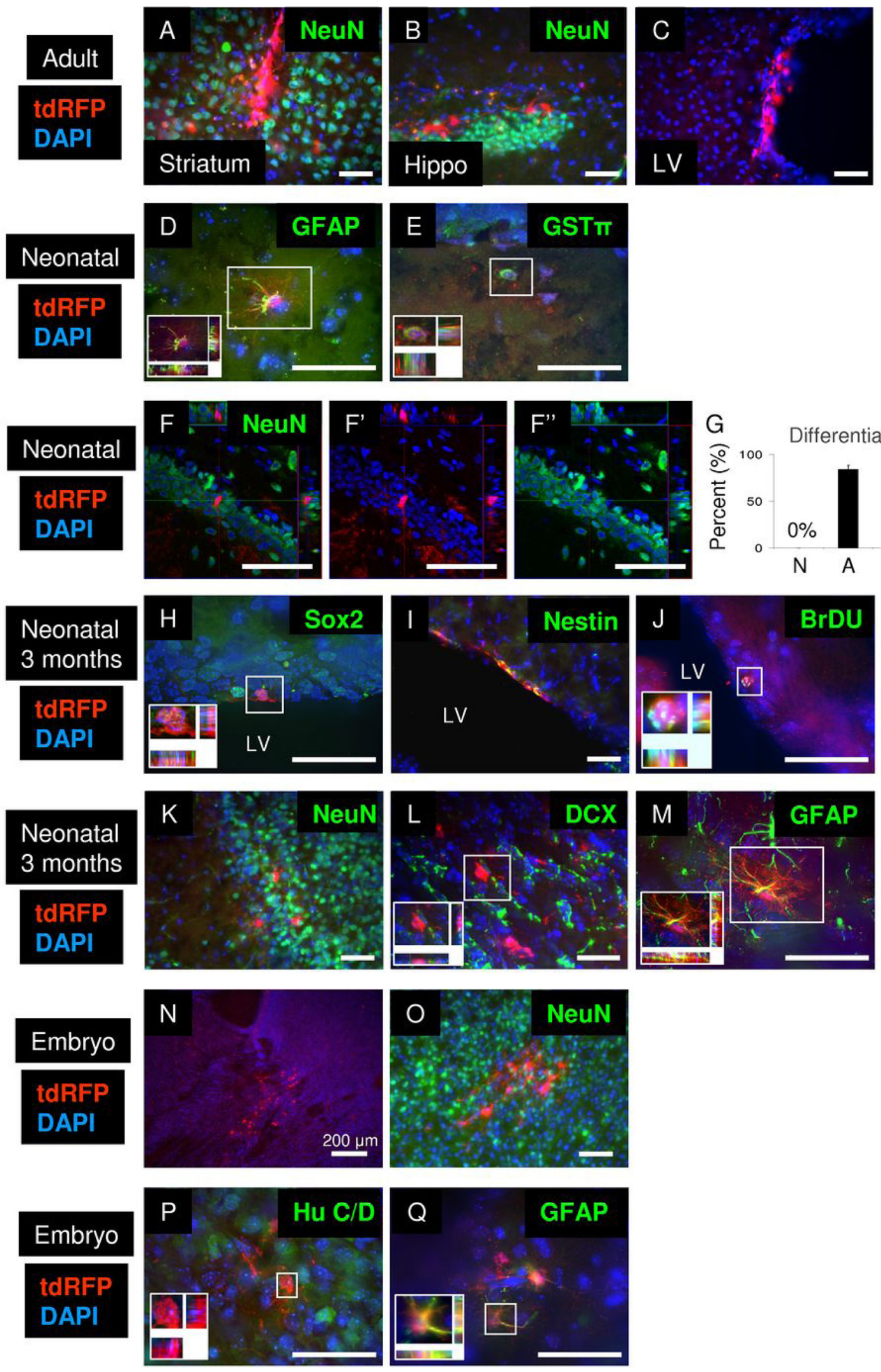

Figure 8. Rad-NSCs differentiate into astrocytes and oligodendrocytes in vivo after transplantation, but not neurons. $\boldsymbol{A}-\boldsymbol{C}$, Transplanted Rad-NSCS (tdRFP, red) did not differentiate into NeuN-positive neurons in the striatum ( $\boldsymbol{A}$, NeuN, green) or hippocampus ( $\boldsymbol{B}$, NeuN, green) in adult brains. $\boldsymbol{C}$, Transplanted Rad-NSCs were observed in the lateral ventricle (LV) in adult brains, but did not migrate in the RMS. $\boldsymbol{D}, \boldsymbol{E}$, Transplanted Rad-NSCs (tdRFP, red) differentiated into GFAP-positive astrocytes ( $\boldsymbol{D}$, green) and GST $\pi$-positive oligodendrocytes ( $\boldsymbol{E}$, green) in the both white and gray matter of neonatal mice after transplantation. $\boldsymbol{F}$, Transplanted Rad-NSCs (red) did not express NeuN (green) in the hippocampus. $\boldsymbol{F}^{\prime}, \boldsymbol{F}^{\prime \prime}$, FITC and TRITC channels, respectively. G, Quantification of Rad-NSC differentiation 1 month after neonatal transplantation into whole brain. $\boldsymbol{H}, \boldsymbol{I}$, Three months after transplantation, tdRFP-positive Rad-NSCs (red) expressed Sox2 ( $\boldsymbol{H}$, green) or Nestin ( $\boldsymbol{I}$, green) in the SVZ. $\boldsymbol{J}$, Three months after transplantation, tdRFP-positive Rad-NSCs (red) stained for BrdU (green) in theSVZ. $K$, L, tdRFP-positive Rad-NSCs (red) that migrated by the RMS did not express NeuN (Figure legend continues.) 
Native reactive astrocytes are unipotent in vivo following stroke

To determine whether endogenous reactive astrocytes are multipotent in the periinfarct area following stroke, we analyzed the cell types that were generated in the peri-infarct area 1 month after stroke. We treated mice with BrdU immediately after stroke surgery for 5 consecutive days and analyzed the mice 1 month after stroke. In agreement with our previous study (Shimada et al., 2010), we did not observe BrdU-positive mature neurons in the cortical peri-infarct area 1 month after stroke ( $n=3$; Fig. 9A). We observed BrdUpositive newly generated astrocytes and oligodendrocytes (Fig. 9B, C), but the origin of these cells was not clear. To determine whether native reactive astrocytes were multipotent in the cortical periinfarct area in vivo after stroke, we performed a lineage tracing study with $G F A P-C r e E R^{T M}$; $t d R F P$ reporter mice. Lineage tracing demonstrated that tdRFPpositive reactive astrocytes did not differentiate into NeuNpositive mature neurons or GST $\pi$-positive oligodendrocytes, but remained as GFAP-positive reactive astrocytes in the peri-infarct area $28 \mathrm{~d}$ following stroke $(n=3$; Fig. $9 D-F)$. Therefore, endogenous tdRFP-positive reactive astrocytes were unipotent and not multipotent in the cortical peri-infarct area following stroke.

\section{Discussion}

Our data demonstrate that cortical GFAP-expressing reactive astrocytes isolated from the stroke injury environment can dedifferentiate into Rad-NSCs that self-renew and are multipotent. Lineage tracing identified reactive astrocytes as a cell of origin for NSCs derived from cortical peri-infarct tissues after stroke. Our data also showed that Rad-NSCs did not originate from cells that had migrated from the SVZ after stroke. Previously, there were several possibilities regarding the cellular origin of cortical neural sphere-forming cells after stroke: (1) neuroblasts or progenitor cells that migrated from the SVZ to the cortex in the absence of stroke, (2) neuroblasts or progenitor cells that migrated from the SVZ to the cortex after stroke, (3) bona fide stem cells that resided in non-neurogenic niches, (4) oligodendrocyte progenitor cells, (5) pericytes, and (6) reactive astrocytes. However, several of the possibilities were unlikely candidates for the origin of Rad-NSCs. For example, in the brains of uninjured adult rodents, NSCs from the SVZ are not known to migrate to the cortex after the completion of development (Chen et al., 2009). In addition, NSCs or progenitors from the SVZ do not migrate to the damaged cortex following brain stab injury or focal cortical stroke (Buffo et al., 2008; Nakagomi et al., 2009; Shimada et al., 2010).

(Figure legend continued.) ( $\boldsymbol{K}$, green) or DCX $(\boldsymbol{L}$, green) in the olfactory bulb 3 months after transplantation. $\boldsymbol{M}$, tdRFP-positive Rad-NSCs (red) differentiated into GFAP-positive astrocytes (green) in the olfactory bulb 3 months after transplantation. $\boldsymbol{N}$, Transplanted Rad-NSCs (red) were observed to engraft in E2O brains. $\mathbf{0}, \boldsymbol{P}$, Transplanted Rad-NSCS (tdRFP, red) did not differentiate into NeuN-positive ( $\boldsymbol{O}$, green) or Hu C/D-positive ( $\boldsymbol{P}$, green) neurons in E20 brains. Q, Transplanted Rad-NSCs (red) expressed GFAP protein in E20 brains. Cell nuclei are shown by DAPI staining (blue). Insets, Magnified deconvolution views of the white boxes. Scale bars: $50 \mu \mathrm{m}$.
Notably, we and others have not been able to generate neural spheres from healthy adult cortical tissue (e.g., contralateral, uninjured side of the cortex after stroke). In our hands, cells that reside in healthy cortex such as NG2-expressing oligodendrocyte progenitor cells, or other adult stem/progenitor cells that potentially reside in non-neurogenic niches, do not form neural spheres in NSC growth medium. Furthermore, Buffo et al. (2008) sorted NG2-positive cells from cortical tissue after stab injury and found that they did not give rise to neural spheres. In agreement with our GFAP lineage tracing studies after stroke, GLASTpositive reactive astrocytes were reported to form multipotential neural spheres in culture after brain stab injury (Buffo et al., 2008). Therefore, reactive astrocytes are the most plausible candidate for the cells that produce neural spheres from cortical tissue, regardless of type of injury. It is noteworthy that we also obtained tdRFP-negative spheres from the peri-infarct area. Although it is of interest to study the origin of these cells, we focused on GFAP-CreER ${ }^{\mathrm{TM}}$-positive cell populations. Because inducible lineage tracing mice do not tag all relevant cells (we found that Cre was not expressed by $100 \%$ of GFAP-positive cells), some of the tdRFP-negative neural spheres could have derived from GFAP-expressing reactive astrocytes that were not labeled. Since reactive astrocytes are phenotypically heterogeneous in the periinfarct area, generating other types of transgenic mice to track subpopulations of reactive astrocytes would likely provide valuable tools to improve understanding of reactive astrogliosis and also possibly neurogenesis (Li and Chopp, 1999; Ganat et al., 2006; Shimada et al., 2010; White et al., 2010; Bi et al., 2011).

Hematopoietic stem cell self-renewal capacity is generally tested in vivo using transplantation studies (Kiel and Morrison, 2008; Nakada et al., 2011). Recently, the Taylor group reported transplantation assays in mice to examine NSC self-renewal capacity (Neumeister et al., 2009). They found that transplantation of typical mouse NSCs into embryonic or neonatal brains could generate neurons (Neumeister et al., 2009). With a similar transplantation model, we showed that cultured PD4-NSCs and adult NSCs that were both derived from the SVZ could migrate to the olfactory bulb in 1 month and produce mature neurons (NeuNpositive). To test the self-renewal and multipotency of Rad- 
NSCs, we transplanted Rad-NSCs into the RMS, SVZ, or SGZ in adult mice and also into the brains of neonatal mice and embryonic mice. Following transplantation, Rad-NSCs in the SVZ expressed Sox 2 and GFAP and BrdU incorporation assays showed that Rad-NSCs proliferated in the SVZ after neonatal transplantation. Transplanted Rad-NSCs could be re-isolated from the SVZ and grown as NSCs, even after remaining $28 \mathrm{~d}$ in vivo. These data suggest that some Rad-NSCs may maintain their capacity to self-renew as multipotent NSCs in the SVZ after transplantation.

Despite their tripotent capacity and ability to produce neurons in culture, Rad-NSCs differed from SVZ-derived PD4-NSCs and adult NSCs in their ability to migrate to the olfactory bulb and also in their ability to generate neurons once they reached the olfactory bulb. The restriction for Rad-NSCs to produce neurons in vivo did not appear to involve age-related changes in environmental (neurogenic) cues from the host; Rad-NSCs did not differentiate into neurons in the brains of adult, neonatal, or embryonic hosts. Transplanted Rad-NSCs were at most, bipotent, producing astrocytes and oligodendrocytes, but not neurons. Based on the similarities between Rad-NSCs and bona fide NSCs from the SVZ in culture, and their differences following transplantation in vivo, Rad-NSCs may provide a valuable system to identify epigenetic and/or other regulators of neurogenesis.

We identified Presenilin 1-based Notch 1 signaling as a mechanism that controlled the generation, proliferation, and selfrenewal of Rad-NSC neural spheres. Consistent with our study, other groups have shown that Notch 1 plays a critical role in the proliferation and self-renewal of embryonic and adult NSCs (Hitoshi et al., 2002; Androutsellis-Theotokis et al., 2006; Breunig et al., 2007; Ables et al., 2010; Borghese et al., 2010; Veeraraghavalu et al., 2010). Therefore, both Rad-NSCs and typical NSCs use a common pathway that regulates stem cells.

The mechanisms that control de-differentiation of reactive astrocytes into Rad-NSCs will be interesting to study. Because we did not obtain any neural spheres from the contralateral side of the cortex after stroke, our data indicate that the cortical periinfarct area contains signals that are permissive to the dedifferentiation of reactive astrocytes. Importantly, however, de-differentiation did not appear to occur in vivo. Lineage tracing demonstrated that GFAP-positive reactive astrocytes were unipotent in the peri-infarct area $28 \mathrm{~d}$ after stroke. Therefore, the combination of signals from the injury environment after stroke (e.g., hypoxia, reactive oxygen species, cytokines, growth factors, Notch ligands, etc.) and signals during culture in NSC growth medium (e.g., bFGF and/or EGF) are both important for the generation of Rad-NSCs.

All reactive astrocytes may not have the capacity to dedifferentiate after stroke injury. Reactive astrocytes are heterogeneous after injury, composed of subpopulations of cells that differentially express proteins associated with stem/progenitor cells (White et al., 2010). Some reactive astrocytes proliferate, but many do not (Robel et al., 2011). We recently identified RC2/ Nestin/GFAP-positive reactive astrocytes in the cortical periinfarct after stroke (Shimada et al., 2010). The RC2-positive subpopulation of reactive astrocytes also expressed Sox 2 and NICD1, proliferated, and was located immediately adjacent to the infarct core. We found that the RC2 antigen was undetectable in uninjured adult cortex or in the contralateral cortex after stroke. RC2 is an antigen commonly used to label radial glial cells during development, and is not expressed by astrocytes in uninjured adults (Misson et al., 1988; Hartfuss et al., 2001; Dromard et al., 2007). We found that cultured RAD-NSCs as well as typical NSCs express RC2. Based on their unique appearance in injured cortical tissue, their proliferation, and their protein expression, the subpopulation of RC2-positive reactive astrocytes may contain the cell of origin for Rad-NSCs. Because neuroblast migration from the SVZ is limited or absent in strokes that do not involve the striatum (Shimada et al., 2010), proliferating RC2positive reactive astrocytes may provide a target cell population for in vivo reprogramming to enhance neurogenesis in cortical peri-infarct tissues after stroke.

\section{References}

Ables JL, Decarolis NA, Johnson MA, Rivera PD, Gao Z, Cooper DC, Radtke F, Hsieh J, Eisch AJ (2010) Notch1 is required for maintenance of the reservoir of adult hippocampal stem cells. J Neurosci 30:10484-10492.

Alexson TO, Hitoshi S, Coles BL, Bernstein A, van der Kooy D (2006) Notch signaling is required to maintain all neural stem cell populations-irrespective of spatial or temporal niche. Dev Neurosci 28:34-48.

Androutsellis-Theotokis A, Leker RR, Soldner F, Hoeppner DJ, Ravin R, Poser SW, Rueger MA, Bae SK, Kittappa R, McKay RD (2006) Notch signalling regulates stem cell numbers in vitro and in vivo. Nature 442:823-826.

Bakondi B, Shimada IS, Perry A, Munoz JR, Ylostalo J, Howard AB, Gregory CA, Spees JL (2009) CD133 identifies a human bone marrow stem/progenitor cell sub-population with a repertoire of secreted factors that protect against stroke. Mol Ther 17:1938-1947.

Barnabé-Heider F, Göritz C, Sabelström H, Takebayashi H, Pfrieger FW, Meletis K, Frisén J (2010) Origin of new glial cells in intact and injured adult spinal cord. Cell Stem Cell 7:470-482.

Batista-Brito R, Close J, Machold R, Fishell G (2008) The distinct temporal origins of olfactory bulb interneuron subtypes. J Neurosci 28:3966-3975.

Bi B, Salmaso N, Komitova M, Simonini MV, Silbereis J, Cheng E, Kim J, Luft S, Ment LR, Horvath TL, Schwartz ML, Vaccarino FM (2011) Cortical glial fibrillary acidic protein-positive cells generate neurons after perinatal hypoxic injury. J Neurosci 31:9205-9221.

Borghese L, Dolezalova D, Opitz T, Haupt S, Leinhaas A, Steinfarz B, Koch P, Edenhofer F, Hampl A, Brustle O (2010) Inhibition of notch signaling in human embryonic stem cell-derived neural stem cells delays G1/S phase transition and accelerates neuronal differentiation in vitro and in vivo. Stem Cells 28:955-964.

Breunig JJ, Silbereis J, Vaccarino FM, Sestan N, Rakic P (2007) Notch regulates cell fate and dendrite morphology of newborn neurons in the postnatal dentate gyrus. Proc Natl Acad Sci U S A 104:20558-20563.

Buffo A, Rite I, Tripathi P, Lepier A, Colak D, Horn AP, Mori T, Götz M (2008) Origin and progeny of reactive gliosis: A source of multipotent cells in the injured brain. Proc Natl Acad Sci U S A 105:3581-3586.

Chen J, Kwon CH, Lin L, Li Y, Parada LF (2009) Inducible site-specific recombination in neural stem/progenitor cells. Genesis 47:122-131.

Chow LM, Zhang J, Baker SJ (2008) Inducible Cre recombinase activity in mouse mature astrocytes and adult neural precursor cells. Transgenic Res 17:919-928.

Clarke L, van der Kooy D (2011) The adult mouse dentate gyrus contains populations of committed progenitor cells that are distinct from subependymal zone neural stem cells. Stem Cells 29:1448-1458.

Dimou L, Simon C, Kirchhoff F, Takebayashi H, Götz M (2008) Progeny of Olig2-expressing progenitors in the gray and white matter of the adult mouse cerebral cortex. J Neurosci 28:10434-10442.

Dromard C, Bartolami S, Deleyrolle L, Takebayashi H, Ripoll C, Simonneau L, Prome S, Puech S, Tran VB, Duperray C, Valmier J, Privat A, Hugnot JP (2007) NG2 and Olig2 expression provides evidence for phenotypic deregulation of cultured central nervous system and peripheral nervous system neural precursor cells. Stem Cells 25:340-353.

Eiraku M, Tohgo A, Ono K, Kaneko M, Fujishima K, Hirano T, Kengaku M (2005) DNER acts as a neuron-specific Notch ligand during Bergmann glial development. Nat Neurosci 8:873-880.

Fortini ME (2002) Gamma-secretase-mediated proteolysis in cell-surfacereceptor signalling. Nat Rev Mol Cell Biol 3:673-684.

Furukawa T, Mukherjee S, Bao ZZ, Morrow EM, Cepko CL (2000) rax, Hes1, and notch1 promote the formation of Muller glia by postnatal retinal progenitor cells. Neuron 26:383-394.

Ganat YM, Silbereis J, Cave C, Ngu H, Anderson GM, Ohkubo Y, Ment LR, Vaccarino FM (2006) Early postnatal astroglial cells produce multilineage precursors and neural stem cells in vivo. J Neurosci 26:8609-8621. 
Ge W, Martinowich K, Wu X, He F, Miyamoto A, Fan G, Weinmaster G, Sun YE (2002) Notch signaling promotes astrogliogenesis via direct CSLmediated glial gene activation. J Neurosci Res 69:848-860.

Gould E, Reeves AJ, Graziano MS, Gross CG (1999) Neurogenesis in the neocortex of adult primates. Science 286:548-552.

Hartfuss E, Galli R, Heins N, Götz M (2001) Characterization of CNS precursor subtypes and radial glia. Dev Biol 229:15-30.

Hitoshi S, Alexson T, Tropepe V, Donoviel D, Elia AJ, Nye JS, Conlon RA, Mak TW, Bernstein A, van der Kooy D (2002) Notch pathway molecules are essential for the maintenance, but not the generation, of mammalian neural stem cells. Genes Dev 16:846-858.

Ihrie RA, Alvarez-Buylla A (2008) Cells in the astroglial lineage are neural stem cells. Cell Tissue Res 331:179-191.

Iso T, Kedes L, Hamamori Y (2003) HES and HERP families: multiple effectors of the Notch signaling pathway. J Cell Physiol 194:237-255.

Kiel MJ, Morrison SJ (2008) Uncertainty in the niches that maintain haematopoietic stem cells. Nat Rev Immunol 8:290-301.

Komitova M, Eriksson PS (2004) Sox-2 is expressed by neural progenitors and astroglia in the adult rat brain. Neurosci Lett 369:24-27.

Kornack DR, Rakic P (2001) Cell proliferation without neurogenesis in adult primate neocortex. Science 294:2127-2130.

Laywell ED, Rakic P, Kukekov VG, Holland EC, Steindler DA (2000) Identification of a multipotent astrocytic stem cell in the immature and adult mouse brain. Proc Natl Acad Sci U S A 97:13883-13888.

Li Y, Chopp M (1999) Temporal profile of nestin expression after focal cerebral ischemia in adult rat. Brain Res 838:1-10.

Luche H, Weber O, Nageswara Rao T, Blum C, Fehling HJ (2007) Faithful activation of an extra-bright red fluorescent protein in "knock-in" Crereporter mice ideally suited for lineage tracing studies. Eur J Immunol 37:43-53.

Misson JP, Edwards MA, Yamamoto M, Caviness VS Jr (1988) Identification of radial glial cells within the developing murine central nervous system: studies based upon a new immunohistochemical marker. Brain Res Dev Brain Res 44:95-108.

Mori T, Tanaka K, Buffo A, Wurst W, Kühn R, Götz M (2006) Inducible gene deletion in astroglia and radial glia-a valuable tool for functional and lineage analysis. Glia 54:21-34.

Morshead CM, van der Kooy D (2004) Disguising adult neural stem cells. Curr Opin Neurobiol 14:125-131.

Nakada D, Levi BP, Morrison SJ (2011) Integrating physiological regulation with stem cell and tissue homeostasis. Neuron 70:703-718.

Nakagomi T, Molnár Z, Nakano-Doi A, Taguchi A, Saino O, Kubo S, Clausen M, Yoshikawa H, Nakagomi N, Matsuyama T (2011) Ischemia-induced neural stem/progenitor cells in the pia mater following cortical infarction. Stem Cells Dev 20:2037-2051.

Nakagomi T, Taguchi A, Fujimori Y, Saino O, Nakano-Doi A, Kubo S, Gotoh A, Soma T, Yoshikawa H, Nishizaki T, Nakagomi N, Stern DM, Matsuyama $\mathrm{T}$ (2009) Isolation and characterization of neural stem/progenitor cells from post-stroke cerebral cortex in mice. Eur J Neurosci 29:1842-1852.

Nedergaard M, Ransom B, Goldman SA (2003) New roles for astrocytes: redefining the functional architecture of the brain. Trends Neurosci 26:523-530.

Neumeister B, Grabosch A, Basak O, Kemler R, Taylor V (2009) Neural progenitors of the postnatal and adult mouse forebrain retain the ability to self-replicate, form neurospheres, and undergo multipotent differentiation in vivo. Stem Cells 27:714-723.

Nunes MC, Roy NS, Keyoung HM, Goodman RR, McKhann G 2nd, Jiang L,
Kang J, Nedergaard M, Goldman SA (2003) Identification and isolation of multipotential neural progenitor cells from the subcortical white matter of the adult human brain. Nat Med 9:439-447.

Palmer TD, Markakis EA, Willhoite AR, Safar F, Gage FH (1999) Fibroblast growth factor-2 activates a latent neurogenic program in neural stem cells from diverse regions of the adult CNS. J Neurosci 19:8487-8497.

Parrish-Aungst S, Shipley MT, Erdelyi F, Szabo G, Puche AC (2007) Quantitative analysis of neuronal diversity in the mouse olfactory bulb. J Comp Neurol 501:825-836.

Patten BA, Sardi SP, Koirala S, Nakafuku M, Corfas G (2006) Notch1 signaling regulates radial glia differentiation through multiple transcriptional mechanisms. J Neurosci 26:3102-3108.

Pekny M, Pekna M (2004) Astrocyte intermediate filaments in CNS pathologies and regeneration. J Pathol 204:428-437.

Reynolds BA, Weiss S (1992) Generation of neurons and astrocytes from isolated cells of the adult mammalian central nervous system. Science 255:1707-1710.

Reynolds BA, Weiss S (1996) Clonal and population analyses demonstrate that an EGF-responsive mammalian embryonic CNS precursor is a stem cell. Dev Biol 175:1-13.

Robel S, Berninger B, Götz M (2011) The stem cell potential of glia: lessons from reactive gliosis. Nat Rev Neurosci 12:88-104.

Satow T, Bae SK, Inoue T, Inoue C, Miyoshi G, Tomita K, Bessho Y, Hashimoto N, Kageyama R (2001) The basic helix-loop-helix gene hesr2 promotes gliogenesis in mouse retina. J Neurosci 21:1265-1273.

Seaberg RM, van der Kooy D (2002) Adult rodent neurogenic regions: the ventricular subependyma contains neural stem cells, but the dentate gyrus contains restricted progenitors. J Neurosci 22:1784-1793.

Sellers DL, Maris DO, Horner PJ (2009) Postinjury niches induce temporal shifts in progenitor fates to direct lesion repair after spinal cord injury. J Neurosci 29:6722-6733.

Shimada IS, Peterson BM, Spees JL (2010) Isolation of locally derived stem/ progenitor cells from the peri-infarct area that do not migrate from the lateral ventricle after cortical stroke. Stroke 41:3552-3560.

Shimada IS, Borders A, Aronshtam A, Spees JL (2011) Proliferating reactive astrocytes are regulated by Notch-1 in the peri-infarct area after stroke. Stroke 42:3231-3237.

Sirko S, Neitz A, Mittmann T, Horvat-Bröcker A, von Holst A, Eysel UT, Faissner A (2009) Focal laser-lesions activate an endogenous population of neural stem/progenitor cells in the adult visual cortex. Brain 132:2252-2264.

Sofroniew MV (2009) Molecular dissection of reactive astrogliosis and glial scar formation. Trends Neurosci 32:638-647.

Sofroniew MV, Vinters HV (2009) Astrocytes: biology and pathology. Acta Neuropathol 19:7-35.

Veeraraghavalu K, Choi SH, Zhang X, Sisodia SS (2010) Presenilin $1 \mathrm{mu}-$ tants impair the self-renewal and differentiation of adult murine subventricular zone-neuronal progenitors via cell-autonomous mechanisms involving notch signaling. J Neurosci 30:6903-6915.

White RE, McTigue DM, Jakeman LB (2010) Regional heterogeneity in astrocyte responses following contusive spinal cord injury in mice. J Comp Neurol 518:1370-1390.

Zawadzka M, Rivers LE, Fancy SP, Zhao C, Tripathi R, Jamen F, Young K, Goncharevich A, Pohl H, Rizzi M, Rowitch DH, Kessaris N, Suter U, Richardson WD, Franklin RJ (2010) CNS-resident glial progenitor/ stem cells produce Schwann cells as well as oligodendrocytes during repair of CNS demyelination. Cell Stem Cell 6:578-590. 